Received: 24 April 2017

Accepted: 28 July 2017

Published online: 29 August 2017

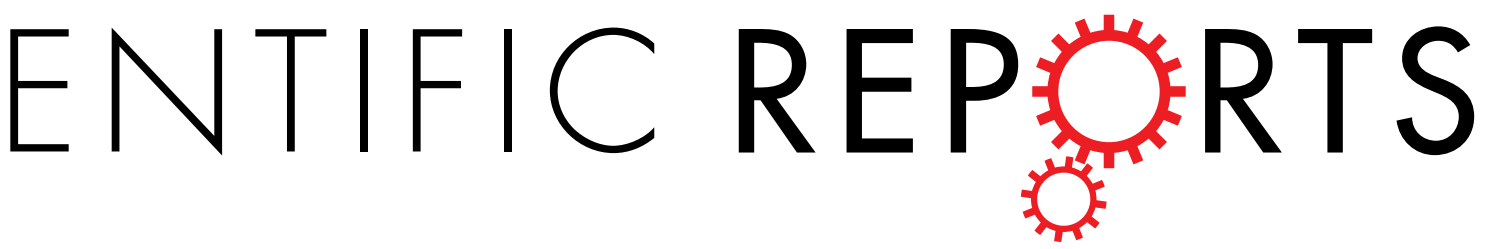

\title{
OPEN Acupuncture for behavioral changes of experimental depressive disorder: a systematic review and meta-analysis
}

Ren-zhong Kou ${ }^{1}$, Hong Chen ${ }^{1}$, Mei-ling $\mathrm{Yu}^{1}$, Tian-cheng $\mathrm{Xu}^{1}$, Shu-ping Fu ${ }^{1,2}$ \& Sheng-feng $\mathrm{Lu}^{1,2}$

Acupuncture is considered to be a promising alternative therapy for depression. Nevertheless, up to now, it remains controversial on the effects exerted by acupuncture on behavioral changes in depression models. Consequently, it's necessary to develop a systematic review and meta-analysis to assess the effect of acupuncture for model rats of depression. Ultimately, 90 studies involving 1861 models were identified. Behavioral indicators including the number of crossings (NC) and the number of rearings (NR) in open field test (OFT), the capacity of sucrose intake (CSI) and the rate of sucrose intake (RSI) in sucrose intake test (SIT), final weight (FW) and gain weight (GW) were employed as main outcomes in depression model rats. The pooled results showed that acupuncture had not less effect than western medicine on improving NC, NR, FW, GW, RSI (P>0.05). However, the CSI improvement was poorer compared with west medicine $(P<0.05)$. In conclusion, acupuncture has not less effect on behavior changes than western medicine, including improving NC, NR, RSI, FW and GW in depression models.

Major Depressive Disorder (MDD) is one of the most common and costly diseases. It is the third most common reason for primary care consultations and will be the second leading cause of disability by 2020 according to the current estimates by $\mathrm{WHO}^{1}$. Nevertheless, the available treatments, especially anti-depressants which are the first-line treatment in medical care, are limited by poor efficacy, lagged therapeutic time and undesirable side effects ${ }^{2}$. New strategies which are more effective and less adverse effects for treating depression are urgently needed.

Acupuncture is widely used in psychiatric conditions' treatment in China for thousands of years ${ }^{3}$. Recent studies demonstrate that depressive patients can benefit from acupuncture therapy in both clinical effectiveness and cost-effectiveness ${ }^{1,4}$. Although confirmed to be a generally safe and well tolerated therapy ${ }^{5}$, the mechanisms underlying the effects of acupuncture on depression have not been fully explained. Therefore, many studies, in which rodents are frequently utilized, have explored mechanisms of antidepressant effects of acupuncture. Given that there were not valid biomarkers accepted as effective links between clinical symptoms and animal end points ${ }^{6}$, depression-like behaviors which simulate the core symptoms in depression are frequently utilized to judge whether the models are successful and whether the interventions are effective ${ }^{3,7-10}$. However, effects of acupuncture on behavioral tests including open field test (OFT), sucrose intake test (SIT), and body weight in depression models are controversial in different studies ${ }^{3,7,8}$. To some extent, these controversial impede the application of these end points.

Due to its potential values, such as promoting the conduct and report of basic researches and providing some guidance to translate the achievements of basic researches to clinical application in acupuncture for depression, and so on, we conducted this systematic review and meta-analysis to evaluate effects of acupuncture on behavioral changes in depression model rats.

${ }^{1}$ The No.2 Clinical Medicine College, Nanjing University of Chinese Medicine, Nanjing, 210023, China. ${ }^{2}$ Key Laboratory of Acupuncture and Medicine Research of Ministry of Education, Nanjing University of Chinese Medicine, Nanjing, 210023, China. Ren-zhong Kou and Hong Chen contributed equally to this work. Correspondence and requests for materials should be addressed to S.-f.L. (email: lushengfeng@njucm.edu.cn) 


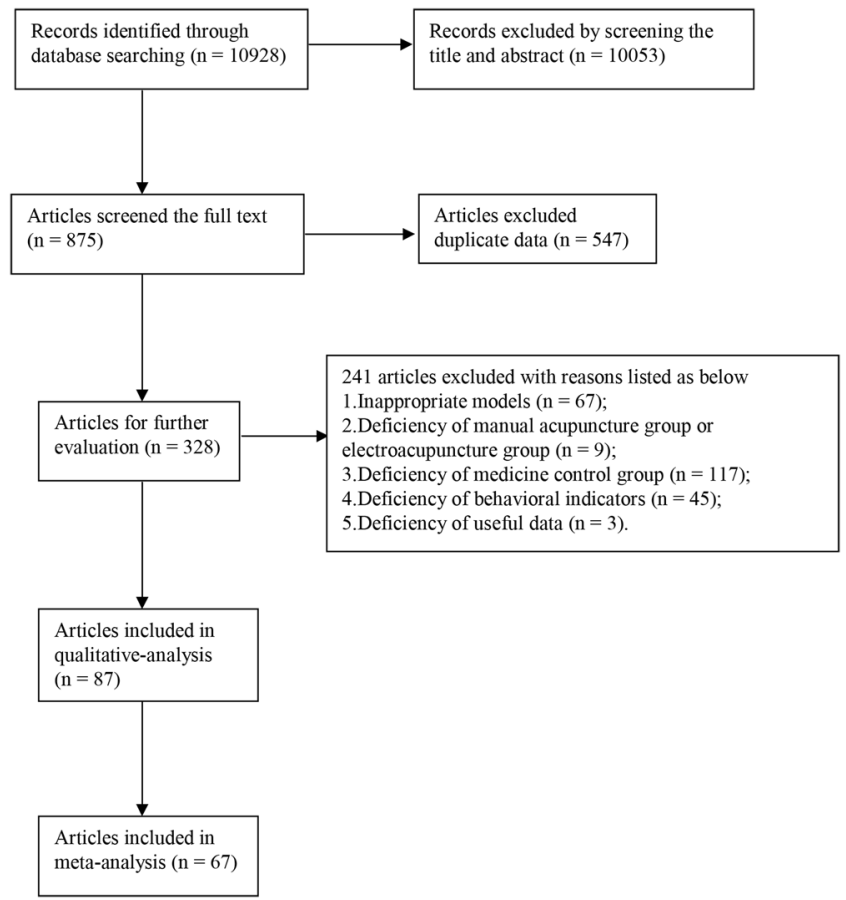

Figure 1. Flowchart of the articles selection process.

\section{Results}

Study inclusion. We screened 10928 potentially relevant articles from seven databases and 10053 articles were excluded by going through titles and abstracts with at least one of the following reasons: (1) the type of study is not animal research (such as case report, clinical trial, review, or meta-analysis), (2) the animal models are not depression models, or the animals in studies are not rats. Full texts of 875 remained articles were assessed for eligibility and 788 of them were excluded according to our inclusion and exclusion criteria. Finally, $87^{11-97}$ articles

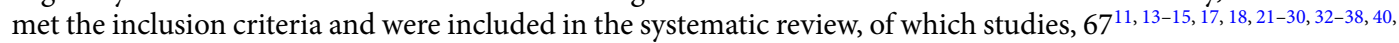
$42,45-47,51,53-56,59-74,76-78,80,81,83-85,87-95,97$ provided raw data were applied in meta-analysis. The process of literature search is displayed in the flowchart (as shown in Fig. 1).

Study characteristics. The characteristics of each research are summarized in Table 1 . We split one article ${ }^{77}$ into three studies and another on ${ }^{39}$ into two studies due to each study selected different depression models. Therefore, 90 studies in 87 articles were included in this review. A majority of studies selected chronic unpredictable mild stress (CUMS) or CUMS combined with solitary models. Besides chronic forced swim stress (CFSS) models ${ }^{11}$, intracerebroventricular injection of ibotenic acid (IA) models ${ }^{22}$, chronic restraint stress (CRS) models ${ }^{26}$, Learned Helpless (LH) models ${ }^{39}$, intracerebroventricular injection of L- $\alpha$-aminoadipic acid (L-AAA) models ${ }^{64}$, intracerebroventricular injection of Excitatory Amino Acid Transporter 1 (EAAT1) antagonist models ${ }^{77}$, prefrontal cortex injection of Excitatory Amino Acid Transporter 2 (EAAT2) antagonist models ${ }^{77}$, pre-shocked animal (PSA) model ${ }^{95}$ were also used in some studies. Totally 1861 depression model rats were included, with the average number of approximately 21 per study, ranging from 14 to 40 . The rats' species included Sprague-Dawley (SD) and Wistar rats. The weight of rats varied between 100-300 g. Eighty-nine studies mentioned randomization and eleven of them selected random number table. None of the studies mentioned blinding. Fifty-eight studies chose intervention and modeling at the same time and the rest chose intervention after or in the process of modeling. Sixty-six studies selected electroacupuncture as intervention and the rest selected manual acupuncture. Only 4 studies did not mention stimulus parameter, the duration of one session varied in $10 \mathrm{~min}, 15 \mathrm{~min}, 20 \mathrm{~min}$, $30 \mathrm{~min}, 40 \mathrm{~min}, 60 \mathrm{~min}$, the number of sessions varied from 5 to 35 and treatment course varied between $5 \mathrm{~d}-42 \mathrm{~d}$. Details of western medicine group and behavioral indicators are also summarized in Table 1 . We also summarized selected acupoints and methods for selection of acupoints in Table S1 and we hope it can be valuable for planning researches in the future.

Study quality and publication bias. The score of each study ranged from 2 to 7 out of a total 10 points quality checklist. Eleven studies got 2 points, forty-one studies got 3 points, twenty-nine studies got 4 points, eight studies got 5 points, one study got 6 points, two studies got 7 points (Table 2). Twenty-four studies utilized anesthetics which have no effect on depressive symptoms. Forty-four studies described the control of temperature, including control of the room and rats' anal temperature. Fifty-six studies were published in peer-reviewed journals. None of the studies described the sample size calculation, allocation concealment, blinded assessment of outcome. Twelve studies described compliance with animal welfare regulations and three studies declared potential conflicts of interest. Only two studies did not describe randomization. 


\begin{tabular}{|c|c|c|c|c|c|c|c|c|c|c|c|c|c|c|c|c|}
\hline Research & Model & Species & Sex & Weight (g) & N & $\begin{array}{l}\text { Rando } \\
\text { mization }\end{array}$ & Blinding & \begin{tabular}{|l|} 
Inter- \\
vention \\
Time
\end{tabular} & $\begin{array}{l}\text { Inter- } \\
\text { ventions }\end{array}$ & Acupoints & \begin{tabular}{|l|} 
Stimulus \\
parameter
\end{tabular} & $\begin{array}{l}\text { Duration } \\
\text { of one } \\
\text { session }\end{array}$ & $\begin{array}{l}\text { The } \\
\text { number of } \\
\text { sessions }\end{array}$ & $\begin{array}{l}\text { Treatment } \\
\text { course }\end{array}$ & Western medicine & Indicators \\
\hline Bao $2014^{11}$ & CFSS & SD rats & Male & $180 \pm 10$ & $10 / 10$ & mention & $\begin{array}{l}\text { not } \\
\text { mention }\end{array}$ & SAME & EA & GV20,GV29 & mention & $10 \mathrm{~min}$ & 14 & $14 \mathrm{~d}$ & Fluoxetine & $\begin{array}{l}\text { OFT:NC,NR; } \\
\text { W:FW; SI:CSI. }\end{array}$ \\
\hline Bao 2014\#12 & CUMS & SD rats & Male & $200 \pm 20$ & $12 / 12$ & mention & $\begin{array}{l}\text { not } \\
\text { mention }\end{array}$ & SAME & EA & GV20,GV29 & mention & $20 \mathrm{~min}$ & 21 & $21 d$ & Fluoxetine & W:FW; \\
\hline Chen $2011^{13}$ & CUMS & SD rats & Female & $180-220$ & $10 / 10$ & $\begin{array}{l}\text { Random } \\
\text { number } \\
\text { table }\end{array}$ & $\begin{array}{l}\text { not } \\
\text { mention }\end{array}$ & $\begin{array}{l}\text { NOT } \\
\text { SAME }\end{array}$ & EA & GV20,GV24 & mention & $30 \mathrm{~min}$ & 14 & $14 \mathrm{~d}$ & Fluoxetine & OFT:NC,NR. \\
\hline $\begin{array}{l}\text { Cheng } \\
2015^{14}\end{array}$ & CUMS & SD rats & Male & 200 & $8 / 8$ & mention & $\begin{array}{l}\text { not } \\
\text { mention }\end{array}$ & SAME & EA & GV20,GV29 & mention & $30 \mathrm{~min}$ & 21 & $21 \mathrm{~d}$ & Fluoxetine & W:FW; SI:CSI. \\
\hline Dai $2010^{15}$ & CUMS & SD rats & Male & $200 \pm 20$ & $13 / 13$ & mention & $\begin{array}{l}\text { not } \\
\text { mention }\end{array}$ & SAME & EA & GV20,GV29 & mention & $20 \mathrm{~min}$ & 21 & $21 \mathrm{~d}$ & Fluoxetine & OFT:NC,NR. \\
\hline Deng $2013^{16}$ & \begin{tabular}{|l} 
CUMS \& \\
Solitary
\end{tabular} & SD rats & Male & $160-180$ & $12 / 12$ & mention & \begin{tabular}{|l|} 
not \\
mention
\end{tabular} & SAME & EA & GV20,GV29 & mention & $20 \mathrm{~min}$ & 21 & $21 \mathrm{~d}$ & Fluoxetine & $\begin{array}{l}\text { OFT:NC,NR; } \\
\text { W:GW; SI:CSI. }\end{array}$ \\
\hline Ding $2016^{17}$ & CUMS & SD rats & Male & $200-210$ & $8 / 8$ & mention & $\begin{array}{l}\text { not } \\
\text { mention }\end{array}$ & $\begin{array}{l}\text { NOT } \\
\text { SAME }\end{array}$ & MA & GV20,GV14 & mention & $20 \mathrm{~min}$ & 18 & $21 d$ & Fluoxetine & \begin{tabular}{|l|} 
OFT:NC,NR; \\
W:FW,GW; SI:RSI.
\end{tabular} \\
\hline Duan $2008^{18}$ & $\begin{array}{l}\text { CUMS \& } \\
\text { Solitary }\end{array}$ & Wistar rats & Male & $150-180$ & $10 / 10$ & mention & \begin{tabular}{|l} 
not \\
mention
\end{tabular} & SAME & EA & $\begin{array}{l}\text { GV20,GV29, } \\
\text { EX-HN1 }\end{array}$ & mention & $20 \mathrm{~min}$ & 21 & $21 d$ & Fluoxetine & $\begin{array}{l}\text { OFT:NC,NR; } \\
\text { W:FW. }\end{array}$ \\
\hline Duan $2016^{19}$ & CUMS & SD rats & Male & $150 \pm 10$ & $16 / 16$ & mention & \begin{tabular}{|l|} 
not \\
mention
\end{tabular} & $\begin{array}{l}\text { NOT } \\
\text { SAME }\end{array}$ & EA & GV20,GV29 & mention & $30 \mathrm{~min}$ & 28 & $28 \mathrm{~d}$ & Fluoxetine & $\begin{array}{l}\text { OFT:NC,NR; } \\
\text { W:FW; }\end{array}$ \\
\hline $\begin{array}{l}\text { Duan } \\
2016 \#^{20}\end{array}$ & $\begin{array}{l}\text { CUMS \& } \\
\text { Solitary }\end{array}$ & Wistar rats & Male & $180-200$ & $10 / 10$ & mention & $\begin{array}{l}\text { not } \\
\text { mention }\end{array}$ & $\begin{array}{l}\text { NOT } \\
\text { SAME }\end{array}$ & EA & GV20,GV29 & mention & $20 \mathrm{~min}$ & 28 & $28 \mathrm{~d}$ & Fluoxetine & $\begin{array}{l}\text { OFT:NC,NR; } \\
\text { W:FW; SI:RSI. }\end{array}$ \\
\hline Fan $2013^{21}$ & $\begin{array}{l}\text { CUMS \& } \\
\text { Solitary }\end{array}$ & SD rats & $\begin{array}{l}\text { Female/ } \\
\text { Male }\end{array}$ & $170-220$ & $10 / 10$ & $\begin{array}{l}\text { Random } \\
\text { number } \\
\text { table }\end{array}$ & $\begin{array}{l}\text { not } \\
\text { mention }\end{array}$ & SAME & MA & $\begin{array}{l}\text { EX-HNl, } \\
\text { PC6,SP6 }\end{array}$ & mention & $30 \mathrm{~min}$ & 21 & $21 d$ & Fluoxetine & $\begin{array}{l}\text { OFT:NC,NR; } \\
\text { |W:FW; SI:CSI. }\end{array}$ \\
\hline Fan $2016^{22}$ & IA & SD rats & Male & $180-220$ & $8 / 8$ & mention & $\begin{array}{l}\text { not } \\
\text { mention }\end{array}$ & $\begin{array}{l}\text { NOT } \\
\text { SAME }\end{array}$ & EA & LI4,LR3 & mention & $15 \mathrm{~min}$ & 21 & $21 d$ & Riluzole & OFT:NC,NR; \\
\hline Fan $2016 \#^{23}$ & CUMS & SD rats & Male & $180-220$ & $8 / 8$ & mention & \begin{tabular}{|l|} 
not \\
mention
\end{tabular} & $\begin{array}{l}\text { NOT } \\
\text { SAME }\end{array}$ & EA & LI4,LR3 & mention & $15 \mathrm{~min}$ & 21 & $21 d$ & Riluzole & $\begin{array}{l}\text { OFT:NC,NR; } \\
\text { W:FW; SI:CSI. }\end{array}$ \\
\hline Fan $2016 * 24$ & CUMS & SD rats & Male & $180-220$ & $8 / 8$ & mention & $\begin{array}{l}\text { not } \\
\text { mention }\end{array}$ & $\begin{array}{l}\text { NOT } \\
\text { SAME }\end{array}$ & MA & LI4,LR3 & $\begin{array}{l}\text { not } \\
\text { mention }\end{array}$ & $\begin{array}{l}\text { not } \\
\text { mention }\end{array}$ & 21 & $21 d$ & Riluzole & $\begin{array}{l}\text { OFT:NC,NR; } \\
\text { W:FW; SI:CSI. }\end{array}$ \\
\hline Fu $2008^{25}$ & CUMS & SD rats & \begin{tabular}{|l|} 
Female/ \\
Male
\end{tabular} & $180-200$ & $8 / 8$ & mention & \begin{tabular}{|l|} 
not \\
mention
\end{tabular} & SAME & EA & LI4,LR3 & mention & $15 \mathrm{~min}$ & 21 & $21 \mathrm{~d}$ & Fluoxetine & $\begin{array}{l}\text { OFT:NC,NR; } \\
\text { W:FW,GW. }\end{array}$ \\
\hline Guo $2016^{26}$ & CRS & SD rats & Male & $200 \pm 20$ & $8 / 8$ & mention & $\begin{array}{l}\text { not } \\
\text { mention }\end{array}$ & SAME & MA & $\begin{array}{l}\text { GV20, } \\
\text { GV29,SP6 }\end{array}$ & mention & $20 \mathrm{~min}$ & 28 & $28 \mathrm{~d}$ & Fluoxetine & $\begin{array}{l}\text { OFT:NC,NR; } \\
\text { W:FW; SI:CSI,RSI. }\end{array}$ \\
\hline $\mathrm{Hu} 2013^{27}$ & $\begin{array}{l}\text { CUMS \& } \\
\text { Solitary }\end{array}$ & SD rats & Male & $200 \pm 20$ & $8 / 8$ & mention & $\begin{array}{l}\text { not } \\
\text { mention }\end{array}$ & SAME & MA & GV20,PC6 & mention & $10 \mathrm{~min}$ & 14 & $28 \mathrm{~d}$ & Fluoxetine & $\begin{array}{l}\text { OFT:NC,NR; } \\
\text { W:FW; SI:RSI }\end{array}$ \\
\hline $\mathrm{Hu} 2014^{28}$ & $\begin{array}{l}\text { CUMS \& } \\
\text { Solitary }\end{array}$ & SD rats & Male & $200 \pm 20$ & $10 / 10$ & \begin{tabular}{|l|}
$\begin{array}{l}\text { Random } \\
\text { number } \\
\text { table }\end{array}$ \\
\end{tabular} & $\begin{array}{l}\text { not } \\
\text { mention }\end{array}$ & SAME & EA & $\begin{array}{l}\text { GV20, } \\
\text { GV29 }\end{array}$ & mention & $30 \mathrm{~min}$ & 28 & $28 \mathrm{~d}$ & Fluoxetine & $\begin{array}{l}\text { OFT:NC,NR; } \\
\text { W:FW; SI:CSI }\end{array}$ \\
\hline $\begin{array}{l}\text { Huang } \\
2005^{29}\end{array}$ & \begin{tabular}{|l} 
CUMS \& \\
Solitary
\end{tabular} & Wistar rats & Male & $200-230$ & $7 / 7$ & mention & \begin{tabular}{|l|} 
not \\
mention
\end{tabular} & $\begin{array}{l}\text { NOT } \\
\text { SAME }\end{array}$ & EA & PC6,CV17 & mention & $30 \mathrm{~min}$ & 21 & $21 \mathrm{~d}$ & Amitriptyline & $\begin{array}{l}\text { OFT:NC,NR; } \\
\text { W:GW; SI:CSI. }\end{array}$ \\
\hline Ji $2013^{30}$ & $\begin{array}{l}\text { CUMS \& } \\
\text { Solitary }\end{array}$ & SD rats & Male & $200 \pm 20$ & $12 / 12$ & mention & $\begin{array}{l}\text { not } \\
\text { mention }\end{array}$ & SAME & EA & $\begin{array}{l}\text { GV20, } \\
\text { GV29 }\end{array}$ & mention & $20 \mathrm{~min}$ & 21 & $21 \mathrm{~d}$ & Fluoxetine & OFT:NC,NR. \\
\hline Jia $2005^{31}$ & $\begin{array}{l}\text { CUMS \& } \\
\text { Solitary }\end{array}$ & SD rats & Male & $160-180$ & $10 / 10$ & mention & $\begin{array}{l}\text { not } \\
\text { mention }\end{array}$ & SAME & EA & $\begin{array}{l}\text { GV20, } \\
\text { GV29 }\end{array}$ & mention & $20 \mathrm{~min}$ & 21 & $21 d$ & Fluoxetine & $\begin{array}{l}\text { OFT:NC,NR; } \\
\text { W:FW; SI:CSI. }\end{array}$ \\
\hline Jiang $2007^{32}$ & CUMS & SD rats & Female & $180-220$ & $8 / 8$ & mention & \begin{tabular}{|l} 
not \\
mention
\end{tabular} & $\begin{array}{l}\text { NOT } \\
\text { SAME }\end{array}$ & EA & $\begin{array}{l}\text { GV20, } \\
\text { GV29 }\end{array}$ & mention & $10 \mathrm{~min}$ & 21 & $21 \mathrm{~d}$ & Fluoxetine & $\begin{array}{l}\text { OFT:NC,NR; } \\
\text { W:FW; SI:CSI. }\end{array}$ \\
\hline Jiang $2013^{33}$ & $\begin{array}{l}\text { CUMS \& } \\
\text { Solitary }\end{array}$ & SD rats & Female & $220-250$ & $18 / 18$ & mention & $\begin{array}{l}\text { not } \\
\text { mention }\end{array}$ & $\begin{array}{l}\text { NOT } \\
\text { SAME }\end{array}$ & EA & LI4,LR3 & mention & $15-20 \mathrm{~min}$ & 14 & $21 \mathrm{~d}$ & Riluzole & $\begin{array}{l}\text { OFT:NC,NR; } \\
\text { W:FW;SI:RSI. }\end{array}$ \\
\hline Jiao $2008^{34}$ & $\begin{array}{l}\text { CUMS \& } \\
\text { Solitary }\end{array}$ & SD rats & Male & $180-200$ & $12 / 12$ & mention & $\begin{array}{l}\text { not } \\
\text { mention }\end{array}$ & SAME & EA & $\begin{array}{l}\text { GV20, } \\
\text { GV29 }\end{array}$ & mention & $30 \mathrm{~min}$ & 21 & $21 d$ & Fluoxetine & $\begin{array}{l}\text { OFT:NC,NR; } \\
\text { W:FW. }\end{array}$ \\
\hline Jin $2015^{35}$ & $\begin{array}{l}\text { CUMS \& } \\
\text { Solitary }\end{array}$ & SD rats & Male & $200 \pm 20$ & $9 / 9$ & mention & $\begin{array}{l}\text { not } \\
\text { mention }\end{array}$ & SAME & MA & $\begin{array}{l}\text { GV20, } \\
\text { PC6 }\end{array}$ & mention & $10 \mathrm{~min}$ & 14 & $28 \mathrm{~d}$ & Fluoxetine & $\begin{array}{l}\text { OFT:NC,NR; } \\
\text { W:FW; SI:CSI. }\end{array}$ \\
\hline Jing $2016^{36}$ & $\begin{array}{l}\text { CUMS \& } \\
\text { Solitary }\end{array}$ & SD rats & Male & $200 \pm 10$ & $8 / 8$ & $\begin{array}{l}\text { random } \\
\text { number } \\
\text { table }\end{array}$ & $\begin{array}{l}\text { not } \\
\text { mention }\end{array}$ & SAME & EA & $\begin{array}{l}\text { GV20, } \\
\text { GV29 }\end{array}$ & mention & $20 \mathrm{~min}$ & 21 & $21 \mathrm{~d}$ & Fluoxetine & OFT:NC,NR; \\
\hline Li $2007^{37}$ & CUMS & SD rats & Male & $200 \pm 20$ & $8 / 8$ & mention & $\begin{array}{l}\text { not } \\
\text { mention }\end{array}$ & SAME & EA & $\begin{array}{l}\text { GV20, } \\
\text { GV29 }\end{array}$ & mention & $20 \mathrm{~min}$ & 21 & $21 d$ & Fluoxetine & $\begin{array}{l}\text { OFT:NC,NR; } \\
\text { W:FW,GW; SI:CSI. }\end{array}$ \\
\hline Li $2008^{38}$ & CUMS & SD rats & Male & $200 \pm 20$ & $13 / 13$ & $\begin{array}{l}\text { Random } \\
\text { number } \\
\text { table }\end{array}$ & $\begin{array}{l}\text { not } \\
\text { mention }\end{array}$ & SAME & EA & $\begin{array}{l}\text { GV20, } \\
\text { GV29 }\end{array}$ & mention & $20 \mathrm{~min}$ & 21 & $21 \mathrm{~d}$ & Fluoxetine & $\begin{array}{l}\text { OFT:NC,NR; } \\
\text { W:FW,GW. }\end{array}$ \\
\hline Li $2011^{39}$ & $\mathrm{LH}$ & $\begin{array}{l}\text { Wistar rats/ } \\
\text { SD rats }\end{array}$ & Male & $200 \pm 20$ & $20 / 15$ & mention & \begin{tabular}{|l} 
not \\
mention
\end{tabular} & $\begin{array}{l}\text { NOT } \\
\text { SAME }\end{array}$ & EA & $\begin{array}{l}\text { GV20, } \\
\text { GB34 }\end{array}$ & mention & $60 \mathrm{~min}$ & 5 & $5 \mathrm{~d}$ & $\begin{array}{l}\text { Chlorimi } \\
\text { pramine }\end{array}$ & OFT:NC,NR. \\
\hline Li $2011 \#^{39}$ & CUMS & $\begin{array}{l}\text { Wistar rats/ } \\
\text { SD rats }\end{array}$ & Male & $200 \pm 20$ & $10 / 10$ & mention & \begin{tabular}{|l} 
not \\
mention
\end{tabular} & $\begin{array}{l}\text { NOT } \\
\text { SAME }\end{array}$ & EA & $\begin{array}{l}\text { GV20, } \\
\text { GB34 }\end{array}$ & \begin{tabular}{|l} 
not \\
mention
\end{tabular} & \begin{tabular}{|l} 
not \\
mention
\end{tabular} & 14 & $28 \mathrm{~d}$ & $\begin{array}{l}\text { Chlorimi } \\
\text { pramine }\end{array}$ & OFT:NC,NR. \\
\hline Li $2011 \&^{40}$ & CUMS & SD rats & Male & $180-220$ & $15 / 15$ & mention & $\begin{array}{l}\text { not } \\
\text { mention }\end{array}$ & $\begin{array}{l}\text { NOT } \\
\text { SAME }\end{array}$ & EA & $\begin{array}{l}\text { GV20, } \\
\text { GV29 }\end{array}$ & mention & $15 \mathrm{~min}$ & 7 & $7 \mathrm{~d}$ & Fluoxetine & $\begin{array}{l}\text { OFT:NC,NR; } \\
\text { W:FW; SI:CSI. }\end{array}$ \\
\hline Li $2014^{41}$ & $\begin{array}{l}\text { CUMS \& } \\
\text { Solitary }\end{array}$ & SD rats & Male & $200 \pm 10$ & $12 / 12$ & mention & $\begin{array}{l}\text { not } \\
\text { mention }\end{array}$ & SAME & EA & $\begin{array}{l}\text { GV20, } \\
\text { GV29 }\end{array}$ & mention & $20 \mathrm{~min}$ & 21 & $21 d$ & Fluoxetine & OFT:NC,NR. \\
\hline Lin $2008^{42}$ & $\begin{array}{l}\text { CUMS \& } \\
\text { Solitary }\end{array}$ & SD rats & Male & $200-230$ & $10 / 10$ & mention & $\begin{array}{l}\text { not } \\
\text { mention }\end{array}$ & SAME & EA & $\begin{array}{l}\text { GV20, } \\
\text { SP6 }\end{array}$ & mention & $20 \mathrm{~min}$ & 21 & $21 d$ & Fluoxetine & $\begin{array}{l}\text { OFT:NC,NR; } \\
\text { W:GW. }\end{array}$ \\
\hline Liu $2005^{43}$ & CUMS & SD rats & Male & $100-120$ & $8 / 8$ & mention & $\begin{array}{l}\text { not } \\
\text { mention }\end{array}$ & $\begin{array}{l}\text { NOT } \\
\text { SAME }\end{array}$ & EA & $\begin{array}{l}\text { GV20, } \\
\text { GB34 }\end{array}$ & mention & $30 \mathrm{~min}$ & 7 & $14 \mathrm{~d}$ & $\begin{array}{l}\text { Chlorimi } \\
\text { pramine }\end{array}$ & $\begin{array}{l}\text { OFT:NC,NR; } \\
\text { W:GW. }\end{array}$ \\
\hline Liu $2008^{44}$ & CUMS & SD rats & Male & $200 \pm 20$ & $8 / 8$ & mention & $\begin{array}{l}\text { not } \\
\text { mention }\end{array}$ & $\begin{array}{l}\text { NOT } \\
\text { SAME }\end{array}$ & EA & $\begin{array}{l}\text { GV20, } \\
\text { GB34 }\end{array}$ & mention & $30 \mathrm{~min}$ & 10 & $21 \mathrm{~d}$ & $\begin{array}{l}\text { Chlorimi } \\
\text { pramine }\end{array}$ & OFT:NC,NR. \\
\hline Liu $2009^{45}$ & $\begin{array}{l}\text { CUMS \& } \\
\text { Solitary }\end{array}$ & SD rats & Male & around 250 & $10 / 10$ & mention & $\begin{array}{l}\text { not } \\
\text { mention }\end{array}$ & SAME & EA & $\begin{array}{l}\text { GV20,GV29, } \\
\text { ST36,SP6 }\end{array}$ & mention & $20 \mathrm{~min}$ & 21 & $21 \mathrm{~d}$ & Fluoxetine & \begin{tabular}{|l|} 
OFT:NC,NR; \\
W:FW,GW SI:RSI
\end{tabular} \\
\hline
\end{tabular}




\begin{tabular}{|c|c|c|c|c|c|c|c|c|c|c|c|c|c|c|c|c|}
\hline Research & Model & Species & Sex & Weight $(\mathrm{g})$ & $\mathrm{N}$ & $\begin{array}{l}\text { Rando } \\
\text { mization }\end{array}$ & Blinding & \begin{tabular}{|l|} 
Inter- \\
vention \\
Time
\end{tabular} & $\begin{array}{l}\text { Inter- } \\
\text { ventions }\end{array}$ & Acupoints & \begin{tabular}{|l|} 
Stimulus \\
parameter
\end{tabular} & $\begin{array}{l}\text { Duration } \\
\text { of one } \\
\text { session }\end{array}$ & \begin{tabular}{|l|}
$\begin{array}{l}\text { The } \\
\text { number of } \\
\text { sessions }\end{array}$ \\
\end{tabular} & $\begin{array}{l}\text { Treatment } \\
\text { course }\end{array}$ & Western medicine & Indicators \\
\hline Liu $2012^{46}$ & CUMS & SD rats & Male & $200-250$ & $8 / 8$ & mention & $\begin{array}{l}\text { not } \\
\text { mention }\end{array}$ & \begin{tabular}{|l|} 
NOT \\
SAME
\end{tabular} & EA & LI4,LR3 & mention & $30 \mathrm{~min}$ & 10 & $21 \mathrm{~d}$ & Fluoxetine & $\begin{array}{l}\text { OFT:NC,NR; } \\
\text { x W:FW; SI:CSI }\end{array}$ \\
\hline Lu $2008^{47}$ & $\begin{array}{l}\text { CUMS \& } \\
\text { Solitary }\end{array}$ & SD rats & Male & $200 \pm 20$ & $8 / 8$ & mention & $\begin{array}{l}\text { not } \\
\text { mention }\end{array}$ & SAME & EA & $\begin{array}{l}\begin{array}{l}\text { GV20,GV29, } \\
\text { ST36,ST40 }\end{array} \\
\end{array}$ & mention & $15 \mathrm{~min}$ & 11 & $22 \mathrm{~d}$ & Maprotiline & $\begin{array}{l}\text { OFT:NC,NR; } \\
\text { W:FW; SI:CSI. }\end{array}$ \\
\hline Lu $2013^{48}$ & CUMS & SD rats & Male & $180-200$ & $10 / 10$ & mention & $\begin{array}{l}\text { not } \\
\text { mention }\end{array}$ & SAME & MA & GV20,PC6 & mention & $10 \mathrm{~min}$ & 14 & $28 \mathrm{~d}$ & Paroxetine & $\begin{array}{l}\text { OFT:NC,NR; } \\
\text { W:FW; SI:RSI. }\end{array}$ \\
\hline Lu $2016^{49}$ & CUMS & SD rats & Male & $180-200$ & $8 / 8$ & mention & $\begin{array}{l}\text { not } \\
\text { mention }\end{array}$ & SAME & MA & GV20,PC6 & mention & $10 \mathrm{~min}$ & 14 & $28 \mathrm{~d}$ & Fluoxetine & $\begin{array}{l}\text { OFT:NC,NR; } \\
\text { W:FW; SI:CSI. }\end{array}$ \\
\hline Lu 2016\# ${ }^{50}$ & CUMS & SD rats & Male & $180-200$ & $8 / 8$ & mention & $\begin{array}{l}\text { not } \\
\text { mention }\end{array}$ & SAME & MA & GV20,PC6 & mention & $10 \mathrm{~min}$ & 14 & $28 \mathrm{~d}$ & Fluoxetine & $\begin{array}{l}\text { OFT:NC,NR; } \\
\text { W:FW; SI:RSI. }\end{array}$ \\
\hline Luo $2016^{51}$ & CUMS & SD rats & Male & $260-300$ & $10 / 10$ & mention & $\begin{array}{l}\text { not } \\
\text { mention }\end{array}$ & \begin{tabular}{|l|} 
NOT \\
SAME
\end{tabular} & EA & LI4,LR3 & mention & $30 \mathrm{~min}$ & 35 & $35 \mathrm{~d}$ & Riluzole & $\begin{array}{l}\text { OFT:NC,NR; } \\
\text { SI:RSI. }\end{array}$ \\
\hline Ma $2016^{52}$ & CUMS & SD rats & Male & $200 \pm 15$ & $12 / 12$ & $\begin{array}{l}\text { random } \\
\text { number } \\
\text { table }\end{array}$ & $\begin{array}{l}\text { not } \\
\text { mention }\end{array}$ & SAME & EA & GV20,GV29 & mention & $20 \mathrm{~min}$ & 21 & $21 \mathrm{~d}$ & Fluoxetine & OFT:NC,NR; \\
\hline Mo $2014^{53}$ & CUMS & SD rats & Male & $200 \pm 10$ & $12 / 12$ & mention & $\begin{array}{l}\text { not } \\
\text { mention }\end{array}$ & SAME & EA & GV20,GV29 & mention & $20 \mathrm{~min}$ & 21 & $21 \mathrm{~d}$ & Fluoxetine & OFT:NC,NR. \\
\hline Pan $2016^{54}$ & CUMS & SD rats & \begin{tabular}{|l|} 
Male/ \\
Female
\end{tabular} & $180-230$ & $10 / 10$ & mention & $\begin{array}{l}\text { not } \\
\text { mention }\end{array}$ & SAME & MA & GV20,PC6,SP6 & mention & $30 \mathrm{~min}$ & 21 & $21 \mathrm{~d}$ & Fluoxetine & $\begin{array}{l}\text { OFT:NC,NR; } \\
\text { W:FW; SI:CSI. }\end{array}$ \\
\hline Qin $2010^{55}$ & $\begin{array}{l}\text { CUMS \& } \\
\text { Solitary }\end{array}$ & SD rats & Male & $170 \pm 10$ & $10 / 10$ & mention & $\begin{array}{l}\text { not } \\
\text { mention }\end{array}$ & SAME & EA & $\begin{array}{l}\text { GV20,GV29, } \\
\text { ST25 }\end{array}$ & mention & $30 \mathrm{~min}$ & 21 & $21 \mathrm{~d}$ & Fluoxetine & $\begin{array}{l}\text { OFT:NC,NR; } \\
\text { W:FW. }\end{array}$ \\
\hline Shao $2016^{56}$ & $\begin{array}{l}\text { CUMS \& } \\
\text { Solitary }\end{array}$ & SD rats & Male & $200 \pm 20$ & $8 / 8$ & mention & $\begin{array}{l}\text { not } \\
\text { mention }\end{array}$ & SAME & MA & GV20,PC6 & mention & $10 \mathrm{~min}$ & 14 & $28 \mathrm{~d}$ & Fluoxetine & $\begin{array}{l}\text { OFT:NC,NR; } \\
\text { W:FW; SI:CSI. }\end{array}$ \\
\hline Shi $2007^{57}$ & CUMS & SD rats & Male & $160-180$ & $10 / 10$ & mention & $\begin{array}{l}\text { not } \\
\text { mention }\end{array}$ & SAME & EA & GV20,GV29 & mention & $20 \min$ & 21 & $21 \mathrm{~d}$ & Fluoxetine & $\begin{array}{l}\text { OFT:NC,NR; } \\
\text { W:FW; SI:CSI. }\end{array}$ \\
\hline Shi $2015^{58}$ & $\begin{array}{l}\text { CUMS \& } \\
\text { Solitary }\end{array}$ & SD rats & Male & $200 \pm 20$ & $10 / 10$ & $\begin{array}{l}\text { random } \\
\text { number } \\
\text { table }\end{array}$ & $\begin{array}{l}\text { not } \\
\text { mention }\end{array}$ & SAME & MA & $\begin{array}{l}\text { GV20,GV29, } \\
\text { GB20,BL23 }\end{array}$ & mention & $30 \min$ & 28 & $28 \mathrm{~d}$ & Fluoxetine & OFT:NC,NR; \\
\hline Song $2014^{59}$ & $\begin{array}{l}\text { CUMS \& } \\
\text { Solitary }\end{array}$ & SD rats & Male & $200 \pm 10$ & $13 / 13$ & mention & $\begin{array}{l}\text { not } \\
\text { mention }\end{array}$ & SAME & EA & GV20,GV29 & mention & $20 \mathrm{~min}$ & 21 & $21 \mathrm{~d}$ & Fluoxetine & $\begin{array}{l}\text { OFT:NC,NR; } \\
\text { W:FW; SI:CSI }\end{array}$ \\
\hline Song $2015^{60}$ & $\begin{array}{l}\text { CUMS \& } \\
\text { Solitary }\end{array}$ & SD rats & Male & $180 \pm 10$ & $8 / 8$ & mention & $\begin{array}{l}\text { not } \\
\text { mention }\end{array}$ & SAME & MA & GV20,GV29 & mention & $20 \min$ & 21 & $21 \mathrm{~d}$ & Fluoxetine & $\begin{array}{l}\text { OFT:NC,NR; } \\
\text { W:FW; SI:CSI. }\end{array}$ \\
\hline Song $2016^{61}$ & $\begin{array}{l}\text { CUMS \& } \\
\text { Solitary }\end{array}$ & SD rats & Male & $200 \pm 20$ & $8 / 8$ & mention & $\begin{array}{l}\text { not } \\
\text { mention }\end{array}$ & SAME & MA & GV20,GV14 & mention & $20 \mathrm{~min}$ & 18 & $21 \mathrm{~d}$ & Fluoxetine & $\begin{array}{l}\text { OFT:NC,NR; } \\
\text { W:FW; SI:RSI. }\end{array}$ \\
\hline Song $2014 \#^{62}$ & $\begin{array}{l}\text { CUMS \& } \\
\text { Solitary }\end{array}$ & SD rats & Male & $200 \pm 10$ & $8 / 8$ & mention & \begin{tabular}{|l} 
not \\
mention
\end{tabular} & SAME & EA & GV20,GV29 & mention & $20 \mathrm{~min}$ & 21 & $21 \mathrm{~d}$ & Fluoxetine & OFT:NC,NR. \\
\hline Sun $2003^{63}$ & CUMS & SD rats & \begin{tabular}{|l|}
$\begin{array}{l}\text { not } \\
\text { mention }\end{array}$ \\
\end{tabular} & $220-240$ & $8 / 8$ & mention & $\begin{array}{l}\text { not } \\
\text { mention }\end{array}$ & $\begin{array}{l}\text { NOT } \\
\text { SAME }\end{array}$ & EA & GV20,ST36 & mention & $30 \mathrm{~min}$ & 21 & $21 \mathrm{~d}$ & $\begin{array}{l}\text { Clomi } \\
\text { pramine }\end{array}$ & OFT:NC,NR. \\
\hline Sun $2013^{64}$ & L-AAA & SD rats & Female & $220-250$ & 9/9 & mention & $\begin{array}{l}\text { not } \\
\text { mention }\end{array}$ & SAME & EA & LI4,LR3 & mention & $15 \mathrm{~min}$ & 21 & $21 \mathrm{~d}$ & Riluzole & $\begin{array}{l}\text { OFT:NC,NR; } \\
\text { W:FW; SI:RSI. }\end{array}$ \\
\hline Sun $2014^{65}$ & $\begin{array}{l}\text { CUMS \& } \\
\text { Solitary }\end{array}$ & SD rats & Male & $200 \pm 20$ & $10 / 10$ & mention & $\begin{array}{l}\text { not } \\
\text { mention }\end{array}$ & SAME & EA & $\begin{array}{l}\text { GV20,GV29, } \\
\text { PC6 }\end{array}$ & mention & $20 \mathrm{~min}$ & 14 & $28 \mathrm{~d}$ & Paroxetine & OFT:NC,NR. \\
\hline Sun $2016^{66}$ & CUMS & SD rats & Male & $200 \pm 20$ & $8 / 8$ & mention & $\begin{array}{l}\text { not } \\
\text { mention }\end{array}$ & $\begin{array}{l}\text { NOT } \\
\text { SAME }\end{array}$ & EA & $\mathrm{CV} 4, \mathrm{ST} 36$ & mention & $30 \mathrm{~min}$ & 14 & $14 \mathrm{~d}$ & Fluoxetine & $\begin{array}{l}\text { OFT:NC,NR; } \\
\text { SI:RSI. }\end{array}$ \\
\hline Tang 2013 & $\begin{array}{l}\text { CUMS \& } \\
\text { Solitary }\end{array}$ & SD rats & Male & $200 \pm 20$ & $10 / 10$ & mention & $\begin{array}{l}\text { not } \\
\text { mention }\end{array}$ & SAME & EA & GV20,GV29 & mention & $20 \mathrm{~min}$ & 21 & $21 \mathrm{~d}$ & Fluoxetine & $\begin{array}{l}\text { OFT:NC,NR; } \\
\text { W:FW; SI:CSI }\end{array}$ \\
\hline Tang $2014^{68}$ & $\begin{array}{l}\text { CUMS \& } \\
\text { Solitary }\end{array}$ & SD rats & Male & $180-220$ & $10 / 10$ & $\begin{array}{l}\text { Random } \\
\text { number } \\
\text { table }\end{array}$ & $\begin{array}{l}\text { not } \\
\text { mention }\end{array}$ & SAME & EA & GV20,GV29 & mention & $20 \mathrm{~min}$ & 21 & $21 \mathrm{~d}$ & Fluoxetine & $\begin{array}{l}\text { OFT:NC,NR; } \\
\text { W:FW; SI:CSI. }\end{array}$ \\
\hline Teng $2013^{69}$ & $\begin{array}{l}\text { CUMS \& } \\
\text { Solitary }\end{array}$ & SD rats & Male & $180 \pm 20$ & $12 / 12$ & mention & $\begin{array}{l}\text { not } \\
\text { mention }\end{array}$ & SAME & EA & GV20,GV29 & mention & $20 \mathrm{~min}$ & 21 & $21 \mathrm{~d}$ & Fluoxetine & $\begin{array}{l}\text { OFT:NC,NR; } \\
\text { W:FW; SI:CSI. }\end{array}$ \\
\hline Wang $2008^{70}$ & $\begin{array}{l}\text { CUMS \& } \\
\text { Solitary }\end{array}$ & SD rats & \begin{tabular}{|l|} 
not \\
mention
\end{tabular} \mid & $160-190$ & $10 / 10$ & \begin{tabular}{|l|} 
Random \\
number \\
table
\end{tabular} & $\begin{array}{l}\text { not } \\
\text { mention }\end{array}$ & $\begin{array}{l}\text { NOT } \\
\text { SAME }\end{array}$ & EA & $\begin{array}{l}\text { GV20,GV29, } \\
\text { SP6 }\end{array}$ & mention & $15 \mathrm{~min}$ & 10 & $21 \mathrm{~d}$ & $\begin{array}{l}\text { Clomi } \\
\text { pramine }\end{array}$ & $\begin{array}{l}\text { OFT:NC,NR; } \\
\text { W:FW,GW; } \\
\text { SI:CSI. }\end{array}$ \\
\hline Wang $2009^{71}$ & CUMS & SD rats & Male & $180-220$ & $10 / 10$ & mention & $\begin{array}{l}\text { not } \\
\text { mention }\end{array}$ & \begin{tabular}{|l|} 
NOT \\
SAME
\end{tabular} & EA & GV20,GV29 & mention & $15 \mathrm{~min}$ & 7 & $7 \mathrm{~d}$ & Fluoxetine & $\begin{array}{l}\text { OFT:NC,NR; } \\
\text { W:FW; SI:CSI. }\end{array}$ \\
\hline Wang $2010^{72}$ & $\begin{array}{l}\text { CUMS \& } \\
\text { Solitary }\end{array}$ & SD rats & Male & $180-220$ & $10 / 10$ & not mention & $\begin{array}{l}\text { not } \\
\text { mention }\end{array}$ & SAME & EA & GV20,LR3 & mention & $20 \mathrm{~min}$ & 21 & $21 \mathrm{~d}$ & Fluoxetine & $\begin{array}{l}\text { OFT:NC,NR; } \\
\text { W:FW; SI:CSI. }\end{array}$ \\
\hline Wang $2013^{73}$ & $\begin{array}{l}\text { CUMS \& } \\
\text { Solitary }\end{array}$ & SD rats & Male & $180-220$ & $12 / 12$ & mention & $\begin{array}{l}\text { not } \\
\text { mention }\end{array}$ & SAME & EA & GV20,GV24 & mention & $15 \mathrm{~min}$ & 7 & $7 \mathrm{~d}$ & Fluoxetine & $\begin{array}{l}\text { OFT:NC,NR; } \\
\text { SI:CSI. }\end{array}$ \\
\hline Wang $2014^{74}$ & $\begin{array}{l}\text { CUMS \& } \\
\text { Solitary }\end{array}$ & SD rats & Male & $180 \pm 20$ & $12 / 12$ & mention & $\begin{array}{l}\text { not } \\
\text { mention }\end{array}$ & $\begin{array}{l}\text { NOT } \\
\text { SAME }\end{array}$ & MA & Ex-B2 & $\begin{array}{l}\text { not } \\
\text { mention }\end{array}$ & $\begin{array}{l}\text { not } \\
\text { mention }\end{array}$ & 28 & $14 \mathrm{~d}$ & Fluoxetine & $\begin{array}{l}\text { OFT:NC,NR; } \\
\text { W:FW. }\end{array}$ \\
\hline Wang $2016^{75}$ & $\begin{array}{l}\text { CUMS \& } \\
\text { Solitary }\end{array}$ & SD rats & Male & $200 \pm 20$ & $12 / 12$ & $\begin{array}{l}\text { random } \\
\text { number } \\
\text { table }\end{array}$ & $\begin{array}{l}\text { not } \\
\text { mention }\end{array}$ & SAME & EA & GV20,GV24 & mention & $20 \mathrm{~min}$ & 21 & $21 \mathrm{~d}$ & Fluoxetine & W:FW; SI:CSI. \\
\hline Wu $2007^{76}$ & CUMS & SD rats & Male & $120-140$ & $10 / 10$ & mention & $\begin{array}{l}\text { not } \\
\text { mention }\end{array}$ & $\begin{array}{l}\text { NOT } \\
\text { SAME }\end{array}$ & EA & ST36 & mention & $15 \mathrm{~min}$ & 22 & $22 \mathrm{~d}$ & Fluoxetine & $\begin{array}{l}\text { OFT:NC,NR; } \\
\text { W:GW. }\end{array}$ \\
\hline Xiao 2014 & $\begin{array}{l}\text { CUMS \& } \\
\text { Solitary }\end{array}$ & SD rats & Male & $250-300$ & $15 / 15$ & mention & $\begin{array}{l}\text { not } \\
\text { mention }\end{array}$ & $\begin{array}{l}\text { NOT } \\
\text { SAME }\end{array}$ & MA & LI4,LR3 & mention & $30 \mathrm{~min}$ & 21 & $35 \mathrm{~d}$ & Riluzole & W:FW; SI:RSI. \\
\hline Xiao 2014\# & EAAT1 & SD rats & Male & $250-300$ & $10 / 10$ & mention & $\begin{array}{l}\text { not } \\
\text { mention }\end{array}$ & \begin{tabular}{|l|} 
NOT \\
SAME
\end{tabular} & MA & LI4,LR3 & mention & $30 \mathrm{~min}$ & 21 & $35 \mathrm{~d}$ & Riluzole & $\begin{array}{l}\text { OFT:NC; } \\
\text { W:FW; SI:RSI. }\end{array}$ \\
\hline $\begin{array}{l}\text { Xiao } \\
2014 \&^{78}\end{array}$ & $\begin{array}{l}\text { CUMS \& } \\
\text { Solitary }\end{array}$ & SD rats & Female & $220-250$ & $20 / 20$ & mention & $\begin{array}{l}\text { not } \\
\text { mention }\end{array}$ & $\begin{array}{l}\text { NOT } \\
\text { SAME }\end{array}$ & EA & LI4,LR3 & $\begin{array}{l}\text { not } \\
\text { mention }\end{array}$ & $\begin{array}{l}\text { not } \\
\text { mention }\end{array}$ & 14 & $21 \mathrm{~d}$ & Riluzole & SI:RSI \\
\hline Xiao $2014^{* 77}$ & EAAT2 & SD rats & Male & $270-290$ & $10 / 10$ & mention & $\begin{array}{l}\text { not } \\
\text { mention }\end{array}$ & $\begin{array}{l}\text { NOT } \\
\text { SAME }\end{array}$ & MA & LI4,LR3 & mention & $30 \mathrm{~min}$ & 21 & $35 \mathrm{~d}$ & Riluzole & $\begin{array}{l}\text { OFT:NC; } \\
\text { W:FW; SI:RSI. }\end{array}$ \\
\hline
\end{tabular}




\begin{tabular}{|c|c|c|c|c|c|c|c|c|c|c|c|c|c|c|c|c|}
\hline Research & Model & Species & Sex & Weight (g) & $\mathbf{N}$ & $\begin{array}{l}\text { Rando } \\
\text { mization }\end{array}$ & Blinding & \begin{tabular}{|l} 
Inter- \\
vention \\
Time
\end{tabular} & $\begin{array}{l}\text { Inter- } \\
\text { ventions }\end{array}$ & Acupoints & $\begin{array}{l}\text { Stimulus } \\
\text { parameter }\end{array}$ & $\begin{array}{l}\text { Duration } \\
\text { of one } \\
\text { session }\end{array}$ & $\begin{array}{l}\text { The } \\
\text { number of } \\
\text { sessions }\end{array}$ & $\begin{array}{l}\text { Treatment } \\
\text { course }\end{array}$ & Western medicine & Indicators \\
\hline $\mathrm{Xu} 2016^{79}$ & $\begin{array}{l}\text { CUMS \& } \\
\text { Solitary }\end{array}$ & SD rats & Male & $200 \pm 20$ & $10 / 10$ & mention & \begin{tabular}{|l} 
not \\
mention
\end{tabular} & SAME & EA & GV20,GV29 & mention & $30 \mathrm{~min}$ & 28 & $28 \mathrm{~d}$ & Fluoxetine & OFT:NC,NR; \\
\hline Xu 2016 $\#^{80}$ & $\begin{array}{l}\text { CUMS \& } \\
\text { Solitary }\end{array}$ & SD rats & Male & $180 \pm 20$ & $8 / 8$ & mention & \begin{tabular}{|l} 
not \\
mention
\end{tabular} & SAME & MA & GV20,GV29 & mention & $10 \mathrm{~min}$ & 21 & $21 d$ & Fluoxetine & $\begin{array}{l}\text { OFT:NC,NR; } \\
\text { W:FW; SI:CSI. }\end{array}$ \\
\hline Yang $2013^{81}$ & $\begin{array}{l}\text { CUMS \& } \\
\text { Solitary }\end{array}$ & SD rats & Male & $200 \pm 20$ & $10 / 10$ & mention & \begin{tabular}{|l|} 
not \\
mention
\end{tabular} & SAME & EA & ST36 & mention & $20 \mathrm{~min}$ & 21 & $21 d$ & Fluoxetine & $\begin{array}{l}\text { OFT:NC,NR; } \\
\text { W:GW; SI:CSI. }\end{array}$ \\
\hline Yang 2013\# $\#^{82}$ & $\begin{array}{l}\text { CUMS \& } \\
\text { Solitary }\end{array}$ & SD rats & Male & $200-220$ & $8 / 8$ & mention & \begin{tabular}{|l} 
not \\
mention
\end{tabular} & \begin{tabular}{|l|} 
NOT \\
SAME
\end{tabular} & EA & GV20,GV29 & mention & $30 \mathrm{~min}$ & 18 & $21 \mathrm{~d}$ & Citalopram & $\begin{array}{l}\text { OFT:NC,NR; } \\
\text { W:FW; SI:RSI. }\end{array}$ \\
\hline Yu $2006^{83}$ & CUMS & SD rats & Male & $120-150$ & $8 / 8$ & mention & \begin{tabular}{|l|} 
not \\
mention
\end{tabular} & \begin{tabular}{|l|} 
NOT \\
SAME
\end{tabular} & EA & GV20,GB34 & mention & $30 \mathrm{~min}$ & 7 & $14 \mathrm{~d}$ & Clomipramine & OFT:NC,NR. \\
\hline Yu $2012^{84}$ & \begin{tabular}{|l} 
CUMS \& \\
Solitary
\end{tabular} & SD rats & Male & $160-180$ & $10 / 10$ & mention & $\begin{array}{l}\text { not } \\
\text { mention }\end{array}$ & SAME & EA & GV20,GV29 & mention & \begin{tabular}{|l|} 
not \\
mention
\end{tabular} & 21 & $21 \mathrm{~d}$ & Fluoxetine & \begin{tabular}{|l|} 
OFT:NC,NR; \\
W:FW; SI:CSI.
\end{tabular} \\
\hline Yu $2016^{85}$ & $\begin{array}{l}\text { CUMS \& } \\
\text { Solitary }\end{array}$ & SD rats & Male & $180 \pm 10$ & $8 / 8$ & mention & \begin{tabular}{|l|} 
not \\
mention
\end{tabular} & SAME & MA & GV20,GV29 & mention & $10 \mathrm{~min}$ & 21 & $21 d$ & Fluoxetine & $\begin{array}{l}\text { OFT:NC,NR; } \\
\text { W:FW; SI:CSI. }\end{array}$ \\
\hline Yu 2006\# $\#^{86}$ & CUMS & SD rats & Male & $200-250$ & $10 / 10$ & not mention & $\begin{array}{l}\text { not } \\
\text { mention }\end{array}$ & \begin{tabular}{|l|} 
NOT \\
SAME
\end{tabular} & EA & GV20, Anmian & mention & $40 \mathrm{~min}$ & 18 & $42 \mathrm{~d}$ & Chlorimipramine & W:FW; SI:CSI,RSI \\
\hline \begin{tabular}{|l|} 
Zhang \\
$2005^{87}$
\end{tabular} & CUMS & SD rats & Male & $180-220$ & $10 / 10$ & mention & \begin{tabular}{|l|} 
not \\
mention
\end{tabular} & \begin{tabular}{|l|} 
NOT \\
SAME
\end{tabular} & MA & $\begin{array}{l}\text { GV20,PC6, } \\
\text { GV24,SP6 }\end{array}$ & mention & $10 \mathrm{~min}$ & 21 & $21 d$ & Fluoxetine & $\begin{array}{l}\text { OFT:NC,NR; } \\
\text { W:FW; SI:CSI. }\end{array}$ \\
\hline \begin{tabular}{|l|} 
Zhang \\
$2008^{88}$
\end{tabular} & $\begin{array}{l}\text { CUMS \& } \\
\text { Solitary }\end{array}$ & SD rats & Male & $160-200$ & $10 / 10$ & mention & \begin{tabular}{|l|} 
not \\
mention
\end{tabular} & SAME & EA & GV20,GV29 & mention & $30 \mathrm{~min}$ & 21 & $21 \mathrm{~d}$ & Fluoxetine & $\begin{array}{l}\text { OFT:NC,NR; } \\
\text { SI:CSI. }\end{array}$ \\
\hline $\begin{array}{l}\text { Zhang } \\
2016^{89}\end{array}$ & CUMS & SD rats & Male & $180-200$ & $10 / 10$ & mention & $\begin{array}{l}\text { not } \\
\text { mention }\end{array}$ & SAME & MA & GV20,GV29 & mention & $10 \mathrm{~min}$ & 21 & $21 \mathrm{~d}$ & Fluoxetine & $\begin{array}{l}\text { OFT:NC,NR; } \\
\text { SI:CSI. }\end{array}$ \\
\hline $\begin{array}{l}\text { Zhang\# } \\
2016^{90}\end{array}$ & CUMS & SD rats & $\begin{array}{l}\text { Male/ } \\
\text { Female }\end{array}$ & $220-290$ & $20 / 20$ & mention & $\begin{array}{l}\text { not } \\
\text { mention }\end{array}$ & SAME & MA & GV20,PC6,SP6 & mention & $30 \mathrm{~min}$ & 21 & $21 \mathrm{~d}$ & Fluoxetine & $\begin{array}{l}\text { OFT:NC,NR; } \\
\text { W:FW; SI:CSI. }\end{array}$ \\
\hline \begin{tabular}{|l|} 
Zhang \\
$2016 \&^{91}$
\end{tabular} & $\begin{array}{l}\text { CUMS \& } \\
\text { Solitary }\end{array}$ & SD rats & Male & $220-270$ & $12 / 12$ & mention & $\begin{array}{l}\text { not } \\
\text { mention }\end{array}$ & \begin{tabular}{|l|} 
NOT \\
SAME
\end{tabular} & EA & LI4,LR3 & mention & $30 \mathrm{~min}$ & 14 & $21 \mathrm{~d}$ & Riluzole & $\begin{array}{l}\text { OFT:NC,NR; } \\
\text { W:FW; SI:RSI. }\end{array}$ \\
\hline $\begin{array}{l}\text { Zhang } \\
2016^{* 92}\end{array}$ & $\begin{array}{l}\text { CUMS \& } \\
\text { Solitary }\end{array}$ & SD rats & Male & $220-240$ & $10 / 10$ & mention & $\begin{array}{l}\text { not } \\
\text { mention }\end{array}$ & SAME & MA & GV20,GV29 & mention & $10 \mathrm{~min}$ & 28 & $28 \mathrm{~d}$ & Fluoxetine & W:FW; SI:RSI. \\
\hline Zhao $2005^{93}$ & $\begin{array}{l}\text { CUMS \& } \\
\text { Solitary }\end{array}$ & SD rats & Male & $200-230$ & $8 / 8$ & mention & \begin{tabular}{|l|} 
not \\
mention
\end{tabular} & SAME & EA & GV20,SP6 & mention & $20 \mathrm{~min}$ & 21 & $21 d$ & Fluoxetine & $\begin{array}{l}\text { OFT:NC,NR; } \\
\text { W:GW. }\end{array}$ \\
\hline \begin{tabular}{|l|} 
Zheng \\
$2013^{94}$
\end{tabular} & CUMS & Wistar rats & Male & $180-220$ & $16 / 16$ & mention & $\begin{array}{l}\text { not } \\
\text { mention }\end{array}$ & \begin{tabular}{|l|} 
NOT \\
SAME
\end{tabular} & EA & $\begin{array}{l}\text { GV20,GV24, } \\
\text { EX-HN1 }\end{array}$ & mention & $20 \mathrm{~min}$ & 14 & $14 \mathrm{~d}$ & Fluoxetine & OFT:NC \\
\hline Zhou $2008^{95}$ & PSA & Wistar rats & Male & $200 \pm 20$ & $10 / 10$ & mention & $\begin{array}{l}\text { not } \\
\text { mention }\end{array}$ & \begin{tabular}{|l|} 
NOT \\
SAME
\end{tabular} & MA & $\begin{array}{l}\text { GV20,PC6, } \\
\text { GV24,SP6 }\end{array}$ & mention & $10 \mathrm{~min}$ & 14 & $14 \mathrm{~d}$ & Doxepin & OFT:NC,NR. \\
\hline Zhu $2015^{96}$ & CUMS & SD rats & Male & $\begin{array}{l}\text { not } \\
\text { mention }\end{array}$ & $9 / 9$ & mention & $\begin{array}{l}\text { not } \\
\text { mention }\end{array}$ & SAME & EA & GV20, Anmian & mention & $30 \mathrm{~min}$ & 14 & $28 \mathrm{~d}$ & Chlorimipramine & $\begin{array}{l}\text { OFT:NC,NR; } \\
\text { SI:RSI. }\end{array}$ \\
\hline \begin{tabular}{|l|} 
Zhuang \\
$2007^{97}$
\end{tabular} & \begin{tabular}{|l|} 
CUMS \& \\
Solitary
\end{tabular} & SD rats & $\begin{array}{l}\text { Female/ } \\
\text { Male }\end{array}$ & $220-290$ & $12 / 12$ & mention & $\begin{array}{l}\text { not } \\
\text { mention }\end{array}$ & SAME & MA & $\begin{array}{l}\text { GV20,BL15, } \\
\text { BL18 }\end{array}$ & mention & $15 \mathrm{~min}$ & 21 & $21 d$ & Fluoxetine & $\begin{array}{l}\text { OFT:NC,NR; } \\
\text { W:FW; SI:CSI. }\end{array}$ \\
\hline
\end{tabular}

Table 1. Characteristics of the studies included. Notes: CFSS: chronic forced swim stress model; CRS: chronic restraint stress model; CUMS: chronic unpredictable mild stress model; CSI:capacity of sucrose intake; EA:electroacupuncture; EAAT1 model:lateral ventricle injection excitatory amino acid transporter 1 antagonists model; EAAT2 model: prefrontal cortex injection excitatory amino acid transporter 2 antagonists model; FW: final weight; GW:gain weight; IA model:intracerebroventricular injection of ibotenic acid model; L-AAA:lateral ventricle injection L- $\alpha$-aminoadipic acid model; LH: learned helpless model; MA: manual acupuncture; NC: the number of crossings; NOT SAME: intervention after or in the process of modeling. NR: the number of rearings; OFT:open field test; PSA: pre-shocked animals; RSI:rate of sucrose intake; SAME:intervention and modeling at the same time;SI:sucrose intake; W:weight;

Outcome and effect estimates. Based on various behavior indicators of the included studies, different pooled data of 69 studies in 67 articles were applied in meta-analysis and 21 studies in 20 articles were not applied in meta-analysis because they did not provide raw data. The data lacked details like observing time or specifications were also not pooled in the analysis because it indicated that the data is not precise enough. All continuous data were presented as mean $\pm \mathrm{SD}$.

The number of crossings (NC) in open field test (OFT): fifty-five studies were pooled in the meta-analysis of NC. The result (Fig. 2) showed that acupuncture had not less effect than western medicine on improving NC in depression model rats $\left(\mathrm{n}=1096, \mathrm{SMD}=-0.09,95 \% \mathrm{CI}=[-0.33\right.$ to 0.15$], \mathrm{P}=0.46$; heterogeneity: $\mathrm{Chi}^{2}=187.50$, $\left.\mathrm{df}=54, \mathrm{P}<0.00001, \mathrm{I}^{2}=71 \%\right)$. Ten studies reported $\mathrm{NC}$ was not pooled in the meta-analysis for the lack of observing time or specification.

The number of rearings (NR) in open field test (OFT): sixty studies were pooled in the meta-analysis of NR. The result (Fig. 3) showed that acupuncture had not less effect than western medicine on improving NR in depression model rats $\left(\mathrm{n}=1202, \mathrm{SMD}=-0.08,95 \% \mathrm{CI}=[-0.24\right.$ to 0.08$], \mathrm{P}=0.0002$; heterogeneity: $\mathrm{Chi}^{2}=104.90$, $\left.\mathrm{df}=59, \mathrm{P}=0.0002, \mathrm{I}^{2}=44 \%\right)$. Two studies reported NR were not pooled in the meta-analysis for the lack of observing time.

Final weight (FW): forty-four studies were pooled in the meta-analysis of FW. The result (Fig. 4) showed that acupuncture had not less effect than western medicine on improving FW of depression model rats $(\mathrm{n}=911$, $\mathrm{SMD}=-0.06,95 \% \mathrm{CI}=[-0.22$ to 0.11$], \mathrm{P}=0.51$; heterogeneity: $\left.\mathrm{Chi}^{2}=65.81, \mathrm{df}=43, \mathrm{P}=0.01, \mathrm{I}^{2}=35 \%\right)$. 


\begin{tabular}{|c|c|c|c|c|c|c|c|c|c|c|c|}
\hline Research & (1) & $(2)$ & (3) & (4) & (5) & $(6)$ & (7) & (8) & (9) & (10) & Total \\
\hline Fan $2016^{* 24}$ & $\sqrt{ }$ & $x$ & $\sqrt{ }$ & $x$ & $x$ & $\sqrt{ }$ & $\sqrt{ }$ & $\sqrt{ }$ & $\sqrt{ }$ & $\sqrt{ }$ & 7 \\
\hline Zhang $2016^{89}$ & $\sqrt{ }$ & $x$ & $\sqrt{ }$ & $x$ & $x$ & $\sqrt{ }$ & $\sqrt{ }$ & $\sqrt{ }$ & $\sqrt{ }$ & $\sqrt{ }$ & 7 \\
\hline Duan 2016 $\#^{20}$ & $\sqrt{ }$ & $x$ & $\sqrt{ }$ & $x$ & $x$ & $x$ & $\sqrt{ }$ & $\sqrt{ }$ & $\sqrt{ }$ & $\sqrt{ }$ & 6 \\
\hline Duan $2016^{19}$ & $\sqrt{ }$ & $x$ & $\sqrt{ }$ & $x$ & $x$ & $x$ & $\sqrt{ }$ & $\sqrt{ }$ & $\sqrt{ }$ & $x$ & 5 \\
\hline Jing $2016^{36}$ & $\sqrt{ }$ & $x$ & $\sqrt{ }$ & $x$ & $x$ & $\sqrt{ }$ & $\sqrt{ }$ & $\times$ & $\sqrt{ }$ & $x$ & 5 \\
\hline Lu $2016 \#^{50}$ & $\sqrt{ }$ & $x$ & $\sqrt{ }$ & $x$ & $x$ & $x$ & $\sqrt{ }$ & $\sqrt{ }$ & $\sqrt{ }$ & $x$ & 5 \\
\hline Mo $2014^{53}$ & $\sqrt{ }$ & $x$ & $\sqrt{ }$ & $x$ & $x$ & $\sqrt{ }$ & $\sqrt{ }$ & $x$ & $\sqrt{ }$ & $x$ & 5 \\
\hline Shi $2015^{58}$ & $\sqrt{ }$ & $x$ & $\sqrt{ }$ & $x$ & $x$ & $x$ & $\sqrt{ }$ & $\sqrt{ }$ & $\sqrt{ }$ & $x$ & 5 \\
\hline Sun $2016^{66}$ & $\sqrt{ }$ & $x$ & $\sqrt{ }$ & $x$ & $x$ & $\sqrt{ }$ & $\sqrt{ }$ & $x$ & $\sqrt{ }$ & $x$ & 5 \\
\hline Tang $2013^{67}$ & $\sqrt{ }$ & $x$ & $\sqrt{ }$ & $x$ & $x$ & $\sqrt{ }$ & $\sqrt{ }$ & $\times$ & $\sqrt{ }$ & $x$ & 5 \\
\hline Wu $2007^{76}$ & $\sqrt{ }$ & $x$ & $\sqrt{ }$ & $x$ & $x$ & $\sqrt{ }$ & $\sqrt{ }$ & $x$ & $\sqrt{ }$ & $\times$ & 5 \\
\hline Cheng $2015^{14}$ & $\sqrt{ }$ & $x$ & $\sqrt{ }$ & $x$ & $x$ & $\sqrt{ }$ & $\sqrt{ }$ & $x$ & $x$ & $x$ & 4 \\
\hline Dai $2010^{15}$ & $\sqrt{ }$ & $x$ & $\sqrt{ }$ & $x$ & $x$ & $\sqrt{ }$ & $x$ & $x$ & $\sqrt{ }$ & $x$ & 4 \\
\hline Deng $2013^{16}$ & $\sqrt{ }$ & $x$ & $\sqrt{ }$ & $x$ & $x$ & $\sqrt{ }$ & $\sqrt{ }$ & $x$ & $x$ & $x$ & 4 \\
\hline Ding $2016^{17}$ & $\sqrt{ }$ & $x$ & $\sqrt{ }$ & $x$ & $x$ & $x$ & $\sqrt{ }$ & $x$ & $\sqrt{ }$ & $x$ & 4 \\
\hline Duan $2008^{18}$ & $\sqrt{ }$ & $x$ & $\sqrt{ }$ & $x$ & $x$ & $\sqrt{ }$ & $x$ & $x$ & $\sqrt{ }$ & $x$ & 4 \\
\hline Hu $2013^{27}$ & $\sqrt{ }$ & $x$ & $\sqrt{ }$ & $x$ & $x$ & $\sqrt{ }$ & $\sqrt{ }$ & $x$ & $x$ & $x$ & 4 \\
\hline Huang $2005^{29}$ & $\sqrt{ }$ & $x$ & $\sqrt{ }$ & $x$ & $x$ & $\sqrt{ }$ & $\sqrt{ }$ & $x$ & $x$ & $x$ & 4 \\
\hline Li $2008^{38}$ & $\sqrt{ }$ & $x$ & $\sqrt{ }$ & $x$ & $x$ & $\sqrt{ }$ & $x$ & $x$ & $\sqrt{ }$ & $x$ & 4 \\
\hline Li $2014^{41}$ & $\sqrt{ }$ & $x$ & $\sqrt{ }$ & $x$ & $x$ & $x$ & $\sqrt{ }$ & $x$ & $\sqrt{ }$ & $x$ & 4 \\
\hline Liu $2009^{45}$ & $\sqrt{ }$ & $x$ & $\sqrt{ }$ & $x$ & $x$ & $x$ & $\sqrt{ }$ & $x$ & $\sqrt{ }$ & $x$ & 4 \\
\hline Liu $2012^{46}$ & $\sqrt{ }$ & $x$ & $\sqrt{ }$ & $x$ & $x$ & $x$ & $\sqrt{ }$ & $x$ & $\sqrt{ }$ & $x$ & 4 \\
\hline Lu $2008^{47}$ & $\sqrt{ }$ & $x$ & $\sqrt{ }$ & $x$ & $x$ & $\sqrt{ }$ & $x$ & $x$ & $\sqrt{ }$ & $x$ & 4 \\
\hline Lu $2013^{48}$ & $\sqrt{ }$ & $x$ & $\sqrt{ }$ & $x$ & $x$ & $x$ & $x$ & $\sqrt{ }$ & $\sqrt{ }$ & $x$ & 4 \\
\hline Luo $2016^{51}$ & $\sqrt{ }$ & $x$ & $\sqrt{ }$ & $x$ & $x$ & $\sqrt{ }$ & $x$ & $x$ & $\sqrt{ }$ & $x$ & 4 \\
\hline Ma $2016^{52}$ & $\sqrt{ }$ & $x$ & $\sqrt{ }$ & $x$ & $x$ & $x$ & $\sqrt{ }$ & $\times$ & $\sqrt{ }$ & $x$ & 4 \\
\hline Qin $2010^{55}$ & $\sqrt{ }$ & $x$ & $\sqrt{ }$ & $x$ & $x$ & $x$ & $\sqrt{ }$ & $x$ & $\sqrt{ }$ & $x$ & 4 \\
\hline Song $2016^{61}$ & $\sqrt{ }$ & $x$ & $\sqrt{ }$ & $x$ & $x$ & $x$ & $\sqrt{ }$ & $x$ & $\sqrt{ }$ & $x$ & 4 \\
\hline Song $2014 \#^{62}$ & $\sqrt{ }$ & $x$ & $\sqrt{ }$ & $x$ & $x$ & $\sqrt{ }$ & $x$ & $\times$ & $\sqrt{ }$ & $x$ & 4 \\
\hline Sun $2013^{64}$ & $\sqrt{ }$ & $x$ & $\sqrt{ }$ & $x$ & $x$ & $\sqrt{ }$ & $x$ & $x$ & $\sqrt{ }$ & $x$ & 4 \\
\hline Sun $2014^{65}$ & $\sqrt{ }$ & $x$ & $\sqrt{ }$ & $x$ & $x$ & $x$ & $\sqrt{ }$ & $\times$ & $\sqrt{ }$ & $x$ & 4 \\
\hline Tang $2014^{68}$ & $\sqrt{ }$ & $x$ & $\sqrt{ }$ & $x$ & $x$ & $x$ & $\sqrt{ }$ & $x$ & $\sqrt{ }$ & $x$ & 4 \\
\hline Wang $2016^{75}$ & $\sqrt{ }$ & $x$ & $\sqrt{ }$ & $x$ & $x$ & $\sqrt{ }$ & $x$ & $x$ & $\sqrt{ }$ & $x$ & 4 \\
\hline Xiao $2014 \&^{78}$ & $\sqrt{ }$ & $x$ & $\sqrt{ }$ & $x$ & $x$ & $\sqrt{ }$ & $x$ & $x$ & $\sqrt{ }$ & $x$ & 4 \\
\hline Xu $2016^{79}$ & $\sqrt{ }$ & $x$ & $\sqrt{ }$ & $x$ & $x$ & $x$ & $x$ & $\sqrt{ }$ & $\sqrt{ }$ & $x$ & 4 \\
\hline Yang $2013 \#^{82}$ & $\sqrt{ }$ & $x$ & $\sqrt{ }$ & $x$ & $x$ & $x$ & $x$ & $\sqrt{ }$ & $\sqrt{ }$ & $x$ & 4 \\
\hline Yu $2006 \#^{86}$ & $\sqrt{ }$ & $x$ & $x$ & $x$ & $x$ & $x$ & $\sqrt{ }$ & $\sqrt{ }$ & $\sqrt{ }$ & $x$ & 4 \\
\hline Zhang $2016 \&^{91}$ & $\sqrt{ }$ & $x$ & $\sqrt{ }$ & $x$ & $x$ & $\sqrt{ }$ & $\sqrt{ }$ & $x$ & $x$ & $x$ & 4 \\
\hline Zhang $2016^{* 92}$ & $\sqrt{ }$ & $x$ & $\sqrt{ }$ & $x$ & $x$ & $\sqrt{ }$ & $\sqrt{ }$ & $x$ & $x$ & $x$ & 4 \\
\hline Zhu $2015^{96}$ & $\sqrt{ }$ & $x$ & $\sqrt{ }$ & $x$ & $x$ & $x$ & $\sqrt{ }$ & $x$ & $\sqrt{ }$ & $x$ & 4 \\
\hline Bao $2014^{11}$ & $\sqrt{ }$ & $x$ & $\sqrt{ }$ & $x$ & $x$ & $x$ & $x$ & $x$ & $\sqrt{ }$ & $x$ & 3 \\
\hline Bao 2014\#\# & $\sqrt{ }$ & $x$ & $\sqrt{ }$ & $x$ & $x$ & $x$ & $x$ & $x$ & $\sqrt{ }$ & $x$ & 3 \\
\hline Chen $2011^{13}$ & $\sqrt{ }$ & $x$ & $\sqrt{ }$ & $x$ & $x$ & $x$ & $x$ & $x$ & $\sqrt{ }$ & $x$ & 3 \\
\hline Fan $2016^{22}$ & $\sqrt{ }$ & $x$ & $\sqrt{ }$ & $x$ & $x$ & $x$ & $x$ & $x$ & $\sqrt{ }$ & $x$ & 3 \\
\hline Fan 2016 $\#^{23}$ & $\sqrt{ }$ & $x$ & $\sqrt{ }$ & $x$ & $x$ & $x$ & $x$ & $x$ & $\sqrt{ }$ & $x$ & 3 \\
\hline Guo $2016^{26}$ & $\sqrt{ }$ & $x$ & $\sqrt{ }$ & $x$ & $x$ & $x$ & $\sqrt{ }$ & $\times$ & $x$ & $x$ & 3 \\
\hline Hu $2014^{28}$ & $\sqrt{ }$ & $x$ & $\sqrt{ }$ & $x$ & $x$ & $x$ & $\sqrt{ }$ & $x$ & $x$ & $x$ & 3 \\
\hline Ji $2013^{30}$ & $\sqrt{ }$ & $x$ & $\sqrt{ }$ & $x$ & $x$ & $x$ & $x$ & $x$ & $\sqrt{ }$ & $x$ & 3 \\
\hline Jia $2005^{31}$ & $\sqrt{ }$ & $x$ & $\sqrt{ }$ & $x$ & $x$ & $x$ & $\sqrt{ }$ & $\times$ & $x$ & $x$ & 3 \\
\hline Jiang $2007^{32}$ & $\sqrt{ }$ & $x$ & $\sqrt{ }$ & $x$ & $x$ & $\sqrt{ }$ & $x$ & $\times$ & $x$ & $x$ & 3 \\
\hline Jiao $2008^{34}$ & $\sqrt{ }$ & $x$ & $\sqrt{ }$ & $x$ & $x$ & $x$ & $x$ & $x$ & $\sqrt{ }$ & $x$ & 3 \\
\hline Jin $2015^{35}$ & $\sqrt{ }$ & $x$ & $\sqrt{ }$ & $x$ & $x$ & $x$ & $\sqrt{ }$ & $\times$ & $x$ & $x$ & 3 \\
\hline Li $2007^{37}$ & $\sqrt{ }$ & $x$ & $\sqrt{ }$ & $x$ & $x$ & $x$ & $x$ & $x$ & $\sqrt{ }$ & $x$ & 3 \\
\hline Li $2011^{39}$ & $\sqrt{ }$ & $x$ & $\sqrt{ }$ & $x$ & $x$ & $x$ & $\sqrt{ }$ & $\times$ & $x$ & $x$ & 3 \\
\hline Li 2011\# $\#^{39}$ & $\sqrt{ }$ & $x$ & $\sqrt{ }$ & $x$ & $x$ & $x$ & $\sqrt{ }$ & $x$ & $x$ & $x$ & 3 \\
\hline Li $2011 \&^{40}$ & $\sqrt{ }$ & $x$ & $\sqrt{ }$ & $x$ & $x$ & $x$ & $x$ & $x$ & $\sqrt{ }$ & $x$ & 3 \\
\hline Liu $2005^{43}$ & $\sqrt{ }$ & $x$ & $\sqrt{ }$ & $x$ & $x$ & $x$ & $x$ & $x$ & $\sqrt{ }$ & $x$ & 3 \\
\hline \multicolumn{12}{|l|}{ Continued } \\
\hline
\end{tabular}




\begin{tabular}{|c|c|c|c|c|c|c|c|c|c|c|c|}
\hline Research & (1) & (2) & (3) & $(4)$ & (5) & (6) & (7) & $(8)$ & (9) & $(10)$ & Total \\
\hline Liu $2008^{44}$ & $\sqrt{ }$ & $\times$ & $\sqrt{ }$ & $x$ & $\times$ & $\times$ & $\times$ & $\sqrt{ }$ & $\times$ & $x$ & 3 \\
\hline Lu $2016^{49}$ & $\sqrt{ }$ & $\times$ & $\sqrt{ }$ & $\times$ & $\times$ & $\times$ & $\times$ & $\sqrt{ }$ & $\times$ & $\times$ & 3 \\
\hline Pan $2016^{54}$ & $\sqrt{ }$ & $\times$ & $\sqrt{ }$ & $\times$ & $\times$ & $\times$ & $\sqrt{ }$ & $\times$ & $\times$ & $\times$ & 3 \\
\hline Shao $2016^{56}$ & $\sqrt{ }$ & $\times$ & $\sqrt{ }$ & $x$ & $\times$ & $\times$ & $\sqrt{ }$ & $\times$ & $\times$ & $\times$ & 3 \\
\hline Shi $2007^{57}$ & $\sqrt{ }$ & $\times$ & $\sqrt{ }$ & $x$ & $\times$ & $\times$ & $\times$ & $\times$ & $\sqrt{ }$ & $x$ & 3 \\
\hline Song $2015^{60}$ & $\sqrt{ }$ & $\times$ & $\sqrt{ }$ & $x$ & $\times$ & $\times$ & $\sqrt{ }$ & $\times$ & $\times$ & $x$ & 3 \\
\hline Sun $2003^{63}$ & $\sqrt{ }$ & $\times$ & $\sqrt{ }$ & $x$ & $\times$ & $\times$ & $\times$ & $\times$ & $\sqrt{ }$ & $x$ & 3 \\
\hline Teng $2013^{69}$ & $\sqrt{ }$ & $\times$ & $\sqrt{ }$ & $x$ & $\times$ & $\times$ & $\sqrt{ }$ & $\times$ & $\times$ & $x$ & 3 \\
\hline Wang $2008^{70}$ & $\sqrt{ }$ & $x$ & $\sqrt{ }$ & $x$ & $\times$ & $\times$ & $\times$ & $\times$ & $\sqrt{ }$ & $x$ & 3 \\
\hline Wang $2013^{73}$ & $\sqrt{ }$ & $\times$ & $\sqrt{ }$ & $\times$ & $\times$ & $\times$ & $\times$ & $x$ & $\sqrt{ }$ & $x$ & 3 \\
\hline Wang $2014^{74}$ & $\sqrt{ }$ & $\times$ & $\sqrt{ }$ & $\times$ & $\times$ & $\times$ & $\times$ & $\times$ & $\sqrt{ }$ & $\times$ & 3 \\
\hline Xiao $2014 \#^{77}$ & $\sqrt{ }$ & $\times$ & $\sqrt{ }$ & $\times$ & $\times$ & $\sqrt{ }$ & $\times$ & $\times$ & $\times$ & $x$ & 3 \\
\hline Xu $2016 \#^{80}$ & $\sqrt{ }$ & $\times$ & $\sqrt{ }$ & $\times$ & $\times$ & $\times$ & $\sqrt{ }$ & $x$ & $x$ & $x$ & 3 \\
\hline Yang $2013^{81}$ & $\sqrt{ }$ & $x$ & $\sqrt{ }$ & $x$ & $x$ & $x$ & $x$ & $x$ & $\sqrt{ }$ & $x$ & 3 \\
\hline Yu $2006^{83}$ & $\sqrt{ }$ & $x$ & $\sqrt{ }$ & $\times$ & $\times$ & $\times$ & $x$ & $x$ & $\sqrt{ }$ & $x$ & 3 \\
\hline Yu $2012^{84}$ & $\sqrt{ }$ & $\times$ & $\sqrt{ }$ & $\times$ & $x$ & $\times$ & $\sqrt{ }$ & $\times$ & $x$ & $x$ & 3 \\
\hline Yu $2016^{85}$ & $\sqrt{ }$ & $\times$ & $\sqrt{ }$ & $x$ & $\times$ & $\times$ & $\sqrt{ }$ & $\times$ & $x$ & $x$ & 3 \\
\hline Zhang $2005^{87}$ & $\sqrt{ }$ & $\times$ & $\sqrt{ }$ & $\times$ & $\times$ & $\times$ & $\times$ & $\times$ & $\sqrt{ }$ & $\times$ & 3 \\
\hline Zhang $2008^{88}$ & $\sqrt{ }$ & $\times$ & $\sqrt{ }$ & $\times$ & $\times$ & $\times$ & $\sqrt{ }$ & $\times$ & $\times$ & $x$ & 3 \\
\hline Zhang\# $2016^{90}$ & $\sqrt{ }$ & $\times$ & $\sqrt{ }$ & $x$ & $\times$ & $\times$ & $\sqrt{ }$ & $\times$ & $\times$ & $x$ & 3 \\
\hline Zheng $2013^{94}$ & $\sqrt{ }$ & $\times$ & $\sqrt{ }$ & $\times$ & $\times$ & $\times$ & $x$ & $\times$ & $\sqrt{ }$ & $x$ & 3 \\
\hline Zhou $2008^{95}$ & $\sqrt{ }$ & $x$ & $\sqrt{ }$ & $x$ & $x$ & $\times$ & $x$ & $\times$ & $\sqrt{ }$ & $x$ & 3 \\
\hline Zhuang $2007^{97}$ & $\sqrt{ }$ & $\times$ & $\sqrt{ }$ & $\times$ & $\times$ & $\times$ & $x$ & $\times$ & $\sqrt{ }$ & $x$ & 3 \\
\hline Fan $2013^{21}$ & $\sqrt{ }$ & $\times$ & $\sqrt{ }$ & $x$ & $\times$ & $\times$ & $\times$ & $\times$ & $\times$ & $x$ & 2 \\
\hline Fu $2008^{25}$ & $\sqrt{ }$ & $x$ & $\sqrt{ }$ & $\times$ & $\times$ & $\times$ & $\times$ & $\times$ & $\times$ & $x$ & 2 \\
\hline Jiang $2013^{33}$ & $\sqrt{ }$ & $\times$ & $\sqrt{ }$ & $\times$ & $\times$ & $\times$ & $\times$ & $\times$ & $\times$ & $\times$ & 2 \\
\hline Lin $2008^{42}$ & $\sqrt{ }$ & $\times$ & $\sqrt{ }$ & $\times$ & $\times$ & $\times$ & $\times$ & $\times$ & $\times$ & $\times$ & 2 \\
\hline Song $2014^{59}$ & $\sqrt{ }$ & $\times$ & $\sqrt{ }$ & $\times$ & $\times$ & $\times$ & $\times$ & $\times$ & $\times$ & $x$ & 2 \\
\hline Wang $2009^{71}$ & $\sqrt{ }$ & $x$ & $\sqrt{ }$ & $x$ & $\times$ & $x$ & $\times$ & $\times$ & $\times$ & $x$ & 2 \\
\hline Wang $2010^{72}$ & $\sqrt{ }$ & $\times$ & $\times$ & $\times$ & $\times$ & $\times$ & $\times$ & $\times$ & $\sqrt{ }$ & $x$ & 2 \\
\hline Xiao $2014^{77}$ & $\sqrt{ }$ & $\times$ & $\sqrt{ }$ & $x$ & $\times$ & $\times$ & $\times$ & $\times$ & $\times$ & $x$ & 2 \\
\hline Xiao $2014^{* 77}$ & $\sqrt{ }$ & $x$ & $\sqrt{ }$ & $\times$ & $\times$ & $\times$ & $\times$ & $\times$ & $\times$ & $x$ & 2 \\
\hline Zhao $2005^{93}$ & $\sqrt{ }$ & $x$ & $\sqrt{ }$ & $x$ & $x$ & $x$ & $x$ & $x$ & $x$ & $x$ & 2 \\
\hline
\end{tabular}

Table 2. Assessment of the quality of studies included. Notes: (1) sample size calculation; (2) randomization to treatment group; (3) allocation concealment; (4) blinded assessment of outcome; (5) correctness of methods of modeling; (6) avoidance of anesthetics with resistance to depressive; (7)statements describing control of temperature; (8) compliance with animal welfare regulations; (9) publication in a peer-reviewed journal; (10) declared any potential conflict of interest.

Gain weight (GW): twelve studies were pooled in the meta-analysis of GW. The result (Fig. 5) showed that acupuncture had not less effect than western medicine on improving GW of depression model rats $(n=220$, $\mathrm{SMD}=-0.02,95 \% \mathrm{CI}=[-0.71$ to 0.66$], \mathrm{P}=0.95$; heterogeneity: $\left.\mathrm{Chi}^{2}=58.17, \mathrm{df}=11, \mathrm{P}<0.00001, \mathrm{I}^{2}=81 \%\right)$.

The capacity of sucrose intake (CSI): twenty-nine studies were pooled in the meta-analysis of CSI. The result (Fig. 6) showed that acupuncture had poorer effects than western medicine on improving CSI of depression model rats $\left(\mathrm{n}=568, \mathrm{SMD}=-0.37,95 \% \mathrm{CI}=[-0.71\right.$ to -0.02$], \mathrm{P}=0.04$; heterogeneity: $\mathrm{Chi}^{2}=104.60, \mathrm{df}=28$, $\left.\mathrm{P}<0.00001, \mathrm{I}^{2}=73 \%\right)$. Five studies reported CSI were not pooled in the meta-analysis because of lacking specification.

The rate of sucrose intake (RSI): fifteen studies were pooled in the meta-analysis of RSI. The result (Fig. 7) showed that acupuncture had not less effect than western medicine on improving RSI of depression model rats $\left(\mathrm{n}=332, \mathrm{SMD}=0.16,95 \% \mathrm{CI}=[-0.14\right.$ to 0.45$], \mathrm{P}=0.31$; heterogeneity: $\mathrm{Chi}^{2}=24.73, \mathrm{df}=14, \mathrm{P}=0.04$, $\left.\mathrm{I}^{2}=43 \%\right)$.

Proposed mechanisms. Seventy-six included studies provided detailed descriptions about possible mechanisms of acupuncture in behavior changes of depression models. A summary of proposed mechanisms is shown in Table 3.

Investigation of heterogeneity. Sensitivity analysis: sensitivity analysis showed that heterogeneity reduced to be acceptable after removing two studies, three studies and one study of which coincidence degree was poor among other studies pooled in the meta-analysis of NR, CSI and RSI, respectively. So, to a large extent, these studies may be the origination of heterogeneity in NR, CSI and RSI. Tests for overall effects in these cases showed that acupuncture had not less effect than western medicine on improving $\mathrm{NR}(\mathrm{Z}=1.14(\mathrm{P}=0.25)), \mathrm{CSI}(\mathrm{Z}=1.00$ 


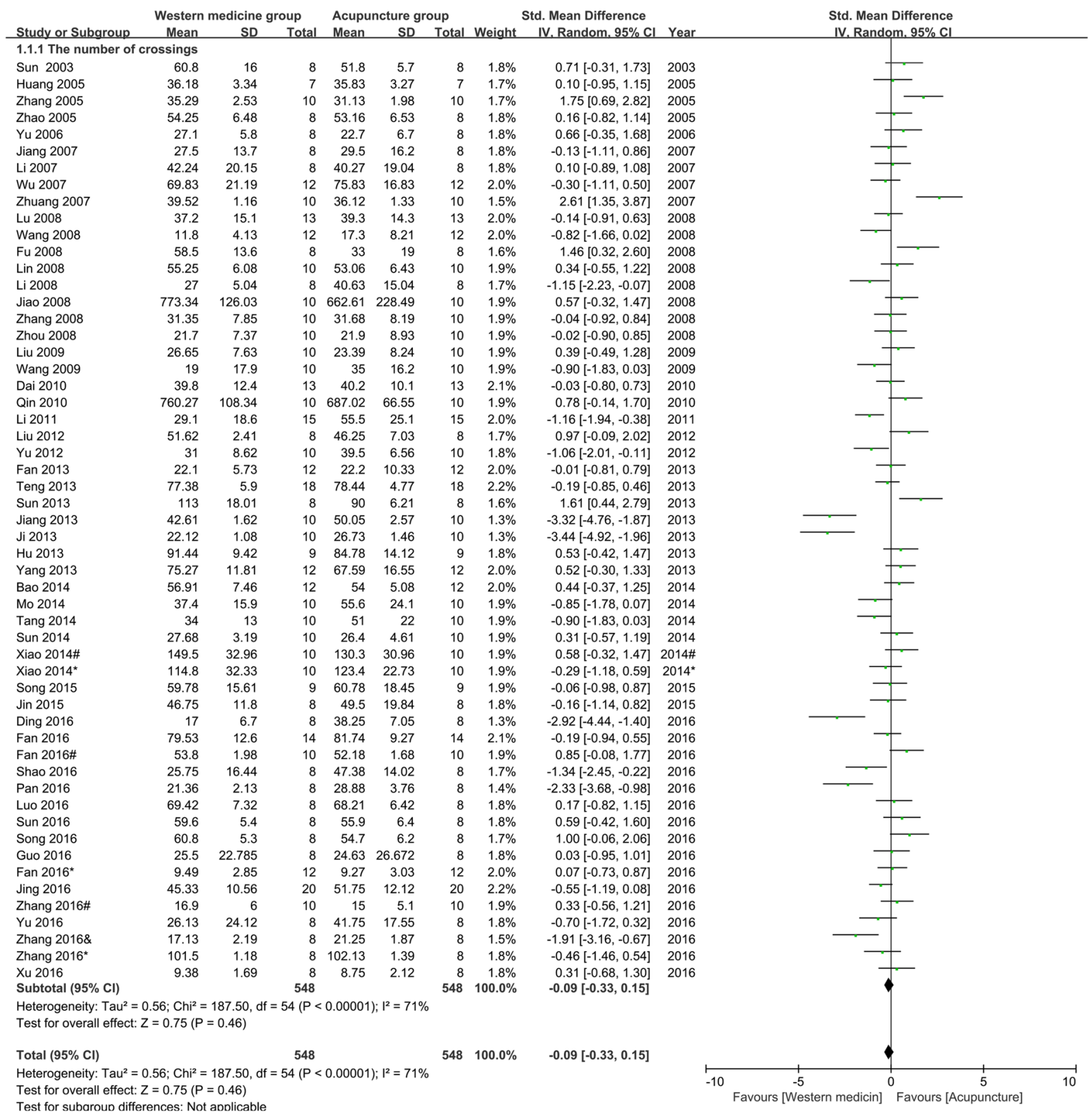

Figure 2. Pool result of acupuncture versus western medicine on NC.

$(\mathrm{P}=0.32))$, RSI $(\mathrm{Z}=0.68(\mathrm{P}=0.50))$ in depression model rats. The heterogeneity was not substantially altered after dismissing any study pooled in the meta-analysis of NC and GW.

Subgroup analysis: to further investigate the source of heterogeneity among the studies pooled in the meta-analysis of NC and GW, a subgroup analysis was conducted. Study characteristics, including different types of acupuncture (acupuncture or electroacupuncture), different stimulation acupoints (scalp acupoints, body acupoints, or scalp acupoints and body acupoints), different intervention time (modeling and intervention at the same time or intervention after or in the process of modeling) and different duration of treatment (more than 21d or less than 14d) are thought to be potential factors affecting the effect of acupuncture. The subgroup analysis was done on the basis of these characteristics. It's failed to subgroup the results by acupuncture method and duration on the basis of GW, since only one study selected manual acupuncture and all the studies' duration were more than $21 \mathrm{~d}$. Unfortunately, we did not find the exact source of heterogeneity among studies by subgroup analysis.

Sensitivity analysis and subgroup analysis were not developed because heterogeneity among studies that were pooled in the meta-analysis of $\mathrm{FW}\left(\mathrm{I}^{2}=35 \%(\mathrm{P}=0.01)\right)$ is acceptable. Details of sensitivity analysis and subgroup analysis were shown in Table 4 and the raw data were shown in Figs S1-S9. 


\begin{tabular}{|c|c|c|c|c|c|c|c|c|c|c|c|c|}
\hline \multirow[b]{2}{*}{ Study or Subgroup } & \multicolumn{3}{|c|}{ Western medicine group } & \multicolumn{3}{|c|}{ Acupuncture group } & \multicolumn{2}{|r|}{ Std. Mean Difference } & \multirow{2}{*}{\multicolumn{4}{|c|}{$\begin{array}{l}\text { Std. Mean Difference } \\
\text { IV. Random. } 95 \% \mathrm{Cl}\end{array}$}} \\
\hline & Mean & SD & Total & Mean & SD & Total & Weight & IV. Random. $95 \% \mathrm{Cl}$ & & & & \\
\hline \multicolumn{13}{|c|}{ 1.2.1 The number of rearings } \\
\hline Sun 2003 & 11.7 & 3.8 & 8 & 11.75 & 5.2 & 8 & $1.5 \%$ & $-0.01[-0.99,0.97]$ & 2003 & & - & \\
\hline Zhao 2005 & 20.37 & 4.69 & 8 & 21.46 & 4.87 & 8 & $1.5 \%$ & $-0.22[-1.20,0.77]$ & 2005 & & - & \\
\hline Huang 2005 & 9.55 & 2.21 & 7 & 9.18 & 2.36 & 7 & $1.4 \%$ & $0.15[-0.90,1.20]$ & 2005 & & & \\
\hline Zhang 2005 & 9.14 & 0.8 & 10 & 9.38 & 0.6 & 10 & $1.7 \%$ & $-0.33[-1.21,0.56]$ & 2005 & & & \\
\hline Yu 2006 & 9.8 & 2.1 & 8 & 8 & 2.3 & 8 & $1.5 \%$ & $0.77[-0.25,1.80]$ & 2006 & & & \\
\hline Zhuang 2007 & 13.5 & 2.52 & 12 & 14.92 & 4.5 & 12 & $1.9 \%$ & $-0.38[-1.18,0.43]$ & 2007 & & & \\
\hline Wu 2007 & 18.12 & 1.28 & 10 & 17.68 & 1.24 & 10 & $1.7 \%$ & $0.33[-0.55,1.22]$ & 2007 & & & \\
\hline Li 2007 & 9.2 & 4.8 & 8 & 9.7 & 5.3 & 8 & $1.5 \%$ & $-0.09[-1.07,0.89]$ & 2007 & & - & \\
\hline Jiang 2007 & 8.88 & 4.78 & 8 & 9.54 & 3.39 & 8 & $1.5 \%$ & $-0.15[-1.13,0.83]$ & 2007 & & E & \\
\hline Lin 2008 & 20.29 & 4.19 & 10 & 21.38 & 3.87 & 10 & $1.7 \%$ & $-0.26[-1.14,0.62]$ & 2008 & & $\leftarrow$ & \\
\hline Zhang 2008 & 10.9 & 3.98 & 10 & 11.9 & 4.53 & 10 & $1.7 \%$ & $-0.22[-1.10,0.66]$ & 2008 & & & \\
\hline Fu 2008 & 2.25 & 1.28 & 8 & 7.75 & 5.04 & 8 & $1.3 \%$ & $-1.41[-2.55,-0.28]$ & 2008 & 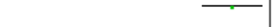 & & \\
\hline Wang 2008 & 8.4 & 1.71 & 10 & 8 & 2.58 & 10 & $1.7 \%$ & $0.18[-0.70,1.05]$ & 2008 & & & \\
\hline Li 2008 & 9.3 & 4.6 & 13 & 9.6 & 4.1 & 13 & $2.0 \%$ & $-0.07[-0.84,0.70]$ & 2008 & & - & \\
\hline Duan 2008 & 10.1 & 2.3 & 10 & 12.5 & 2.1 & 10 & $1.6 \%$ & $-1.04[-1.99,-0.10]$ & 2008 & & & \\
\hline Jiao 2008 & 2.2 & 2.2 & 12 & 3.3 & 2.58 & 12 & $1.9 \%$ & $-0.44[-1.25,0.37]$ & 2008 & & & \\
\hline Lu 2008 & 11.4 & 7.7 & 8 & 6.1 & 3.3 & 8 & $1.4 \%$ & $0.85[-0.19,1.88]$ & 2008 & & & \\
\hline Zhou 2008 & 7.92 & 8.26 & 10 & 11.03 & 6.98 & 10 & $1.7 \%$ & $-0.39[-1.28,0.50]$ & 2008 & & & \\
\hline Liu 2009 & 8.37 & 3.41 & 10 & 9.66 & 4.4 & 10 & $1.7 \%$ & $-0.31[-1.20,0.57]$ & 2009 & & - & \\
\hline Wang 2009 & 6.5 & 4.2 & 10 & 5 & 1.6 & 10 & $1.7 \%$ & $0.45[-0.44,1.34]$ & 2009 & & & \\
\hline Dai 2010 & 10.6 & 3.2 & 13 & 10.3 & 2.2 & 13 & $2.0 \%$ & $0.11[-0.66,0.88]$ & 2010 & & & \\
\hline Wang 2010 & 16.3 & 4.72 & 10 & 17.2 & 3.52 & 10 & $1.7 \%$ & $-0.21[-1.09,0.67]$ & 2010 & & - & \\
\hline Qin 2010 & 11 & 2.56 & 10 & 9.2 & 2.57 & 10 & $1.7 \%$ & $0.67[-0.23,1.58]$ & 2010 & & & \\
\hline Li 2011 & 1.9 & 2.2 & 15 & 4.3 & 4.8 & 15 & $2.1 \%$ & $-0.63[-1.36,0.11]$ & 2011 & & & \\
\hline Liu 2012 & 8.25 & 1.29 & 8 & 9.25 & 1.79 & 8 & $1.5 \%$ & $-0.61[-1.62,0.40]$ & 2012 & & & \\
\hline Yu 2012 & 13 & 3.74 & 10 & 12.5 & 3.44 & 10 & $1.7 \%$ & $0.13[-0.74,1.01]$ & 2012 & & & \\
\hline Hu 2013 & 29.75 & 9.71 & 8 & 23.5 & 3.63 & 8 & $1.4 \%$ & $0.81[-0.23,1.84]$ & 2013 & & & \\
\hline Yang 2013 & 8.16 & 0.96 & 10 & 9.25 & 1.25 & 10 & $1.6 \%$ & $-0.94[-1.87,-0.00]$ & 2013 & & & \\
\hline Ji 2013 & 8.25 & 2.22 & 12 & 8.13 & 2.44 & 12 & $1.9 \%$ & $0.05[-0.75,0.85]$ & 2013 & & - & \\
\hline Sun 2013 & 11.22 & 3.801 & 9 & 10 & 2.389 & 9 & $1.6 \%$ & $0.37[-0.57,1.30]$ & 2013 & & & \\
\hline Fan 2013 & 19.32 & 1.62 & 10 & 23.07 & 2.11 & 10 & $1.3 \%$ & $-1.91[-3.01,-0.81]$ & 2013 & & & \\
\hline Teng 2013 & 10.03 & 2.45 & 12 & 8.19 & 2.46 & 12 & $1.9 \%$ & $0.72[-0.11,1.55]$ & 2013 & & & \\
\hline Jiang 2013 & 10 & 2.62 & 18 & 9.78 & 2.11 & 18 & $2.3 \%$ & $0.09[-0.56,0.74]$ & 2013 & & - & \\
\hline Tang 2013 & 7.93 & 3.16 & 10 & 8.52 & 3.47 & 10 & $1.7 \%$ & $-0.17[-1.05,0.71]$ & 2013 & & E & \\
\hline Hu 2014 & 10.9 & 1.79 & 10 & 11.5 & 2.07 & 10 & $1.7 \%$ & $-0.30[-1.18,0.59]$ & 2014 & & & \\
\hline Sun 2014 & 10 & 4 & 10 & 13 & 4 & 10 & $1.7 \%$ & $-0.72[-1.63,0.19]$ & 2014 & & & \\
\hline Wang 2014 & 13.7 & 2.12 & 12 & 13.9 & 1.43 & 12 & $1.9 \%$ & $-0.11[-0.91,0.69]$ & 2014 & & - & \\
\hline Bao 2014 & 17.3 & 7.69 & 10 & 18.3 & 9.52 & 10 & $1.7 \%$ & $-0.11[-0.99,0.77]$ & 2014 & & - & \\
\hline Tang 2014 & 8.92 & 1.58 & 10 & 8.38 & 1.61 & 10 & $1.7 \%$ & $0.32[-0.56,1.21]$ & 2014 & & & \\
\hline Mo 2014 & 11.44 & 0.8 & 12 & 13.58 & 3.19 & 12 & $1.8 \%$ & $-0.89[-1.73,-0.04]$ & 2014 & & & \\
\hline Song 2014 & 14.92 & 5.52 & 13 & 10.38 & 4.97 & 13 & $1.9 \%$ & $0.84[0.03,1.64]$ & 2014 & & & \\
\hline Song 2014\# & 14.43 & 3.51 & 8 & 12.14 & 2.27 & 8 & $1.5 \%$ & $0.73[-0.29,1.76]$ & 2014\# & & & \\
\hline Song 2015 & 8.63 & 3.02 & 8 & 6.88 & 3.09 & 8 & $1.5 \%$ & $0.54[-0.46,1.55]$ & 2015 & & 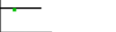 & \\
\hline Jin 2015 & 22.78 & 6.74 & 9 & 16.22 & 6.4 & 9 & $1.5 \%$ & $0.95[-0.04,1.94]$ & 2015 & & & \\
\hline Pan 2016 & 16.67 & 5.2 & 10 & 15.82 & 3.6 & 10 & $1.7 \%$ & $0.18[-0.70,1.06]$ & 2016 & & - & \\
\hline Zhang 2016* & 8.9 & 2.5 & 10 & 9.5 & 2.1 & 10 & $1.7 \%$ & $-0.25[-1.13,0.63]$ & 2016 & & - & \\
\hline Jing 2016 & 9.63 & 1.41 & 8 & 6 & 1.07 & 8 & $0.9 \%$ & $2.74[1.27,4.21]$ & 2016 & & & \\
\hline Shao 2016 & 58.75 & 6.58 & 8 & 61.88 & 5.4 & 8 & $1.5 \%$ & $-0.49[-1.49,0.51]$ & 2016 & & T & \\
\hline Zhang 2016\& & 9.59 & 2.88 & 12 & 8.42 & 3.53 & 12 & $1.9 \%$ & $0.35[-0.46,1.16]$ & 2016 & & & \\
\hline Song 2016 & 6.88 & 2.53 & 8 & 4.88 & 4.29 & 8 & $1.5 \%$ & $0.54[-0.47,1.54]$ & 2016 & & & \\
\hline Yu 2016 & 1 & 1.069 & 8 & 1.63 & 1.685 & 8 & $1.5 \%$ & $-0.42[-1.42,0.57]$ & 2016 & & & \\
\hline Zhang 2016\# & 14.78 & 5.91 & 20 & 19.3 & 4.68 & 20 & $2.3 \%$ & $-0.83[-1.48,-0.18]$ & 2016 & - & & \\
\hline Xu 2016 & 8 & 1.85 & 8 & 8.75 & 1.49 & 8 & $1.5 \%$ & $-0.42[-1.42,0.57]$ & 2016 & & & \\
\hline Sun 2016 & 31.75 & 1.63 & 8 & 34.25 & 1.21 & 8 & $1.2 \%$ & $-1.65[-2.83,-0.47]$ & 2016 & & & \\
\hline Fan 2016\# & 5.4 & 0.9 & 8 & 6.3 & 0.7 & 8 & $1.4 \%$ & $-1.06[-2.12,0.01]$ & 2016 & & & \\
\hline Ding 2016 & 7.25 & 3.54 & 8 & 5.25 & 3.28 & 8 & $1.5 \%$ & $0.55[-0.45,1.56]$ & 2016 & & & \\
\hline Guo 2016 & 8.38 & 3.76 & 8 & 7 & 2.13 & 8 & $1.5 \%$ & $0.43[-0.57,1.42]$ & 2016 & & & \\
\hline Fan 2016* & 7.2 & 1.4 & 8 & 7.8 & 1.5 & 8 & $1.5 \%$ & $-0.39[-1.38,0.60]$ & 2016 & & & \\
\hline Luo 2016 & 9.47 & 3.07 & 14 & 9.83 & 1.45 & 14 & $2.1 \%$ & $-0.15[-0.89,0.60]$ & 2016 & & & \\
\hline Fan 2016 & 12.13 & 0.73 & 8 & 12.41 & 0.93 & 8 & $1.5 \%$ & $-0.32[-1.30,0.67]$ & 2016 & & & \\
\hline Subtotal $(95 \% \mathrm{Cl})$ & & & 601 & & & 601 & $100.0 \%$ & $-0.08[-0.24,0.08]$ & & & & \\
\hline \multicolumn{13}{|c|}{ Heterogeneity: $\mathrm{Tau}^{2}=0.16 ; \mathrm{Chi}^{2}=104.90, \mathrm{df}=59(P=0.0002) ; 1^{2}=44 \%$} \\
\hline Total $(95 \% \mathrm{Cl})$ & & & 601 & & & 601 & $100.0 \%$ & $-0.08[-0.24,0.08]$ & & & & \\
\hline \multicolumn{10}{|c|}{$\begin{array}{l}\text { Heterogeneity: } \text { Tau }^{2}=0.16 ; \mathrm{Chi}^{2}=104.90, \mathrm{df}=59(\mathrm{P}=0.0002) ; \mathrm{I}^{2}=44 \% \\
\text { Test for overall effect: } Z=1.01(P=0.31)\end{array}$} & -5 & & 5 \\
\hline
\end{tabular}

Figure 3. Pool result of acupuncture versus western medicine on NR.

Assessment of publication bias. Funnel plot showed asymmetry and it indicated a potential publication bias (Fig. 8). Begg's test showed there was no significant publication bias $(\mathrm{p}=0.548)$, but the Egger's test $(\mathrm{p}<0.001)$ indicated publication bias possibly existed (Fig. S10). In addition, all of the studies published by

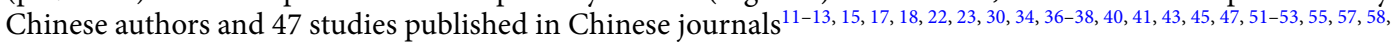
61-68, 70, 72-76,78,79, 81,83, 87, 94-97, 10 studies published in English journals ( 9 of them came from SCI source journals) ${ }^{19}$,

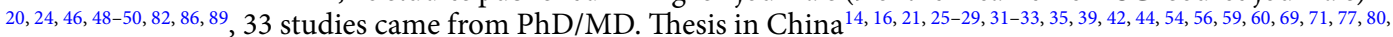
$84,85,88,90-93$

Discussion

Efficacy of acupuncture. To our knowledge, this is the first systematic review and meta-analysis of English and Chinese literatures to investigate the efficacy of acupuncture for animal model on depressive disorder with behavioral changes as the outcome. The present study indicate that compared with western medicine, acupuncture has not less effect on behavior indicators including NC, NR, RSI, FW and GW for depression model rats, but the evidence on whether acupuncture is as effective as western medicine for depression model rats on CSI 


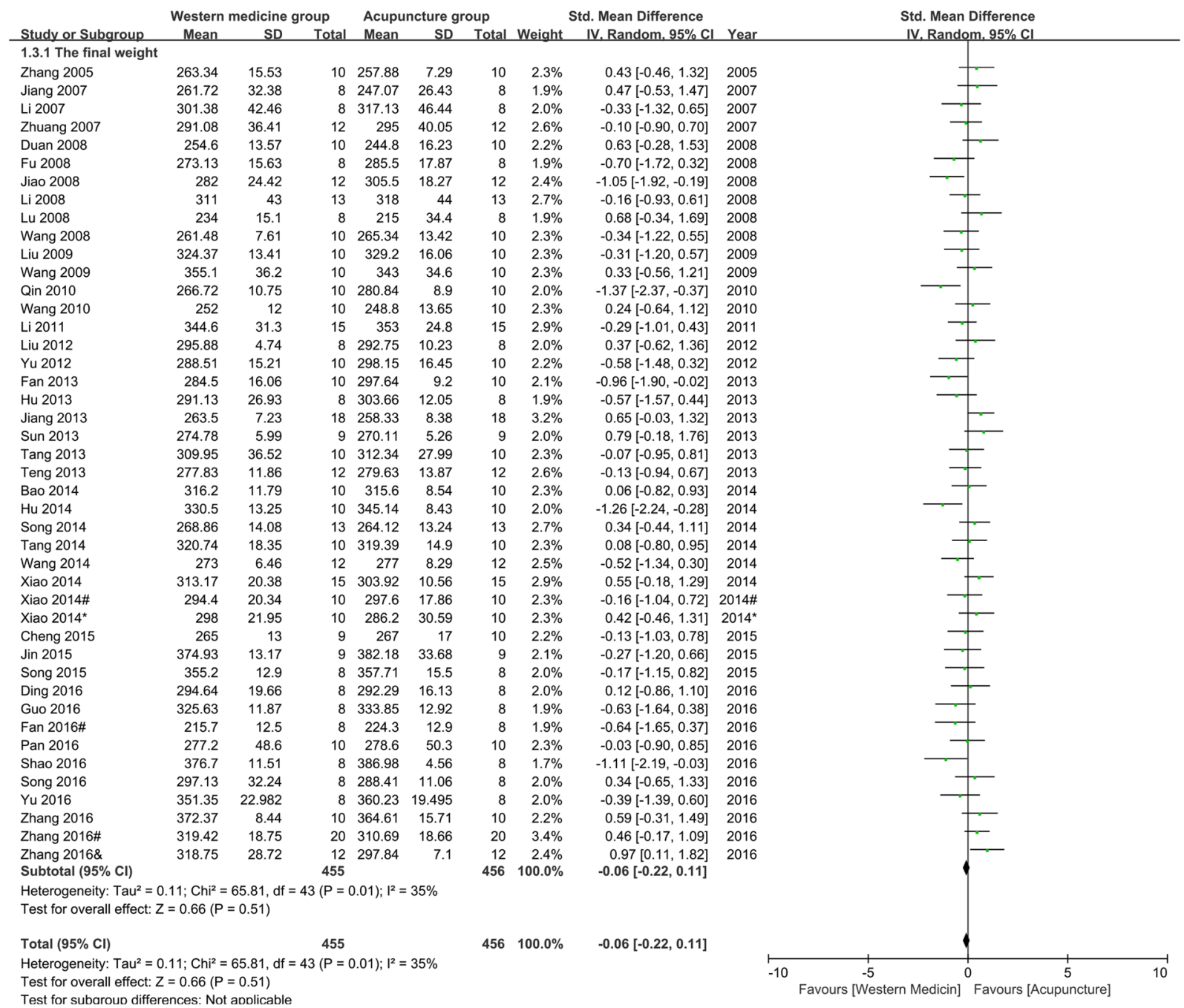

Figure 4. Pool result of acupuncture versus western medicine on FW.

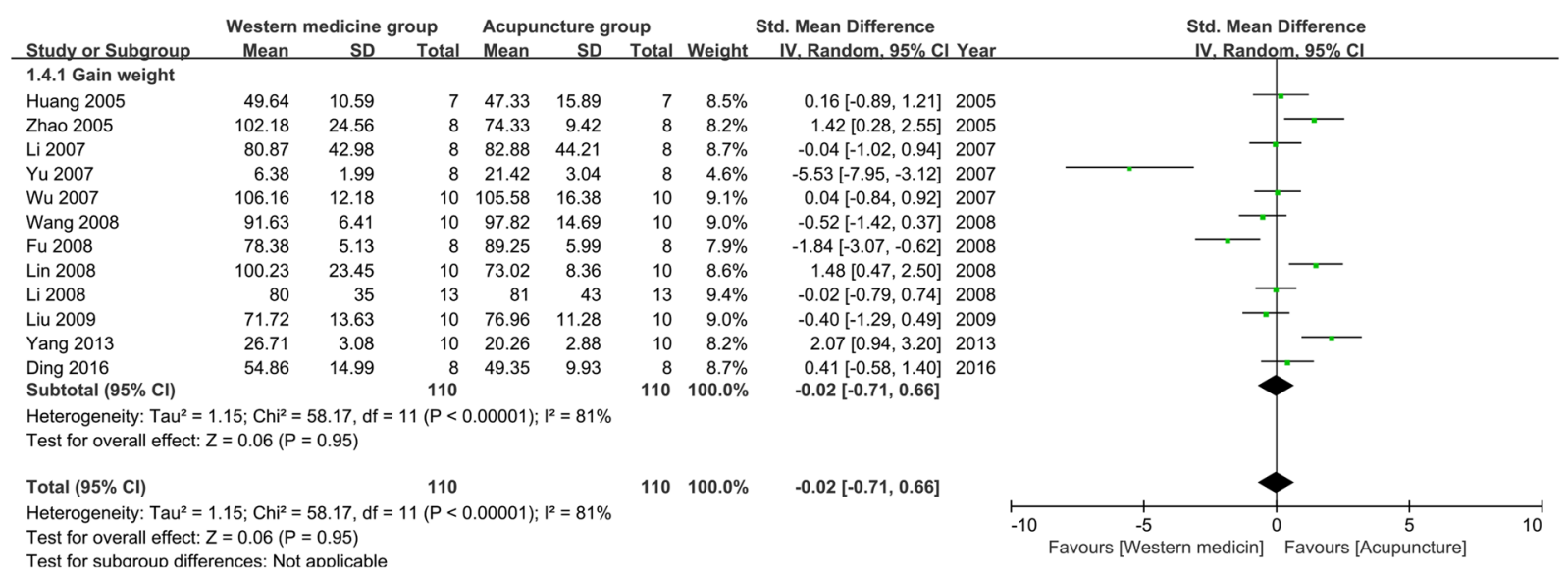

Figure 5. Pool result of acupuncture versus western medicine on GW.

is insufficient. It demonstrates that acupuncture may have a potential disadvantage on improving the appetite of depression model rats in comparison with western medicine. Nevertheless, some researches showed that stimulations to acupoints of the stomach meridian such as Sibai (ST 2), Liangmen (ST 21), and Zusanli (ST 36) were 


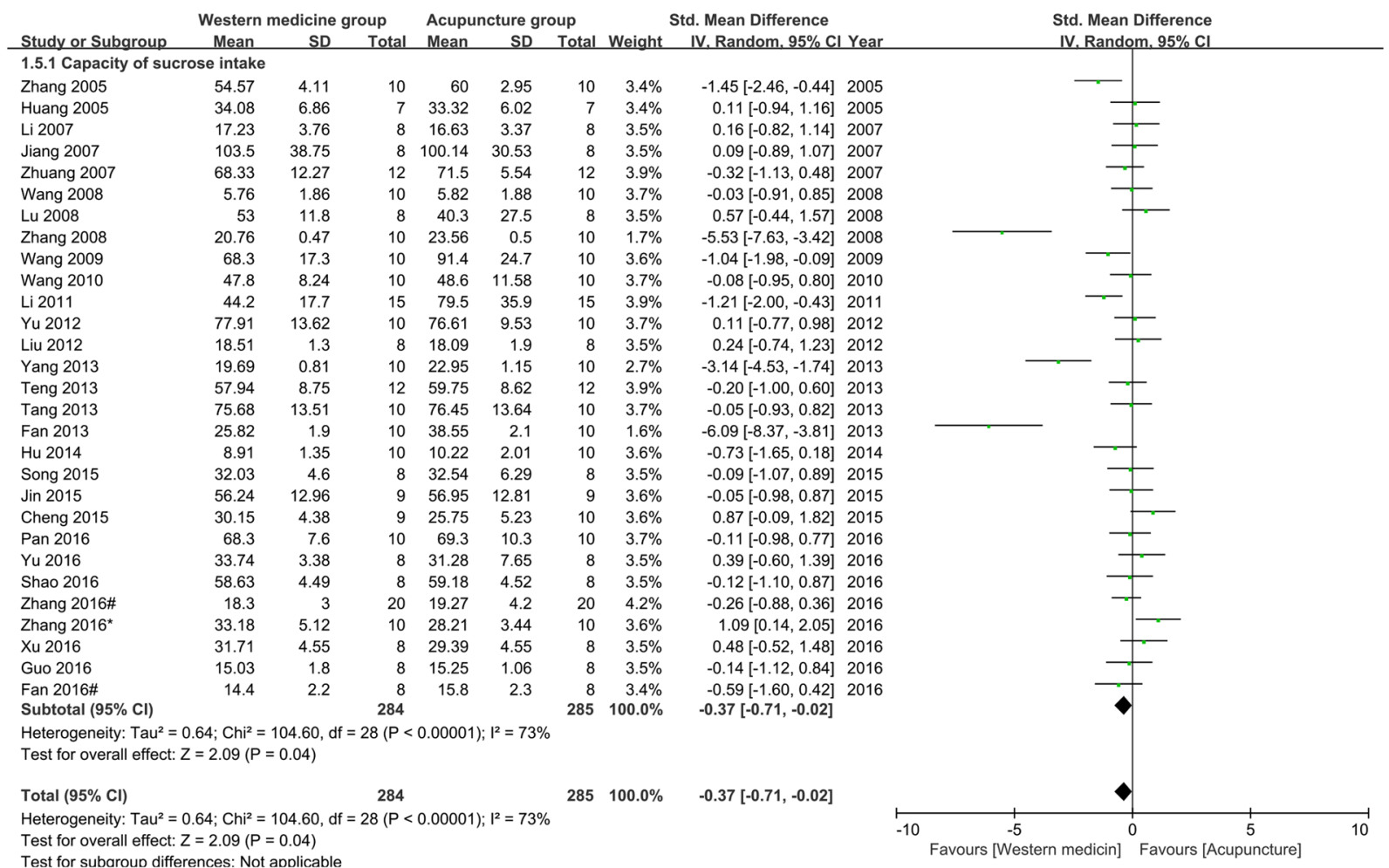

Figure 6. Pool result of acupuncture versus western medicine on CSI.

\begin{tabular}{|c|c|c|c|c|c|c|c|c|c|c|c|c|}
\hline \multirow[b]{2}{*}{ Study or Subgroup } & \multicolumn{3}{|c|}{ Western medicine group } & \multicolumn{3}{|c|}{ Acupuncture group } & \multicolumn{2}{|r|}{ Std. Mean Difference } & \multirow{2}{*}{\multicolumn{4}{|c|}{$\begin{array}{l}\text { Std. Mean Difference } \\
\text { IV. Random. } 95 \% \mathrm{Cl}\end{array}$}} \\
\hline & Mean & SD & Total & Mean & SD & Total & Weight & IV. Random. $95 \% \mathrm{Cl}$ & Year & & om. $95 \% \mathrm{Cl}$ & \\
\hline \multicolumn{13}{|c|}{ 1.6.1 The rate of sucrose intake } \\
\hline Liu 2009 & 78.08 & 13.37 & 10 & 76.63 & 14.44 & 10 & $6.7 \%$ & $0.10[-0.78,0.98]$ & 2009 & & & \\
\hline Sun 2013 & 83.44 & 6.82 & 9 & 84.52 & 4.51 & 9 & $6.2 \%$ & $-0.18[-1.10,0.75]$ & 2013 & & & \\
\hline Jiang 2013 & 83.99 & 2.09 & 18 & 81.54 & 3 & 18 & $8.5 \%$ & $0.93[0.24,1.62]$ & 2013 & & - & \\
\hline Hu 2013 & 86 & 12.85 & 8 & 85.5 & 14.61 & 8 & $5.8 \%$ & $0.03[-0.95,1.01]$ & 2013 & & - & \\
\hline Xiao 2014 & 74.35 & 12.77 & 15 & 77.56 & 10.47 & 15 & $8.2 \%$ & $-0.27[-0.99,0.45]$ & 2014 & & - & \\
\hline Xiao 2014\# & 87.21 & 4.36 & 10 & 79.24 & 17.56 & 10 & $6.5 \%$ & $0.60[-0.30,1.50]$ & 2014\# & & 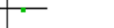 & \\
\hline Xiao 2014\& & 64.68 & 5.43 & 18 & 66.43 & 8.92 & 18 & $8.9 \%$ & $-0.23[-0.89,0.42]$ & $2014 \&$ & & - & \\
\hline Xiao $2014^{*}$ & 86.46 & 7.21 & 10 & 82.36 & 14.14 & 10 & $6.6 \%$ & $0.35[-0.53,1.23]$ & $2014^{*}$ & & \% & \\
\hline Zhang 2016 & 84.34 & 9.39 & 10 & 86.98 & 6.19 & 10 & $6.6 \%$ & $-0.32[-1.20,0.57]$ & 2016 & - & 6 & \\
\hline Ding 2016 & 62.58 & 11.46 & 8 & 71.06 & 12.34 & 8 & $5.6 \%$ & $-0.67[-1.69,0.34]$ & 2016 & & & \\
\hline Zhang 2016\& & 84.5 & 3.92 & 12 & 82.17 & 5.86 & 12 & $7.2 \%$ & $0.45[-0.36,1.26]$ & 2016 & & & \\
\hline Luo 2016 & 82.05 & 8.3 & 14 & 80.76 & 3.33 & 14 & $7.9 \%$ & $0.20[-0.54,0.94]$ & 2016 & & - & \\
\hline Guo 2016 & 85 & 6.1 & 8 & 83 & 5.4 & 8 & $5.8 \%$ & $0.33[-0.66,1.32]$ & 2016 & & - & \\
\hline Sun 2016 & 75.25 & 1.94 & 8 & 71 & 1.73 & 8 & $3.9 \%$ & $2.19[0.87,3.50]$ & 2016 & & & \\
\hline $\begin{array}{l}\text { Song } 2016 \\
\text { Subtotal ( } 95 \% \mathrm{Cl})\end{array}$ & 55.12 & 17.93 & $\begin{array}{r}8 \\
166\end{array}$ & 66.64 & 18.83 & $\begin{array}{r}8 \\
166\end{array}$ & $\begin{array}{r}5.6 \% \\
100.0 \%\end{array}$ & $\begin{array}{l}-0.59[-1.60,0.42] \\
0.16[-0.14,0.45]\end{array}$ & 2016 & & & \\
\hline \multicolumn{13}{|c|}{$\begin{array}{l}\text { Heterogeneity: } \text { Tau }^{2}=0.15 ; \mathrm{Chi}^{2}=24.73, \mathrm{df}=14(P=0.04) ; \mathrm{I}^{2}=43 \% \\
\text { Test for overall effect: } Z=1.03(P=0.31)\end{array}$} \\
\hline Total $(95 \% \mathrm{Cl})$ & & & 166 & & & 166 & $100.0 \%$ & $0.16[-0.14,0.45]$ & & & & \\
\hline \multicolumn{10}{|c|}{ Heterogeneity: $\mathrm{Tau}^{2}=0.15 ; \mathrm{Chi}^{2}=24.73, \mathrm{df}=14(\mathrm{P}=0.04) ; \mathrm{I}^{2}=43 \%$} & -5 & & 10 \\
\hline Test for overall effect & $=1.03(P$ & $0.31)$ & & & & & & & & Favours [Western medicin] & Favours [ & ipuncture] \\
\hline
\end{tabular}

Figure 7. Pool result of acupuncture versus western medicine on RSI.

effective in treating functional dyspepsia ${ }^{98}$, in which poor appetite is one of the core symptoms. In our analysis, Baihui (GV20) and Yintang (GV29) were most frequently used, while acupoints of stomach are scarcely used. Therefore, we consider that acupoints may be the main influencing factor in effecs of acupuncture on symptoms of depression.

Explanations for indicators, models and interventions. In depression research, the OFT is a commonly indicator to assess the general locomotor activity and willingness to explore ${ }^{9}$. The SIT is a behavioral indicator that can assess the degree of anhedonia in models ${ }^{10}$. In addition, obvious change in weight is one of the core depressive-like symptoms. Moreover, some studies had doubt about the effects of acupuncture on these indicators in depression models ${ }^{3,7,8}$, which suggesting whether acupuncture has effects on these indicators needs to be resolved. Therefore, we selected NC, NR, CSI, RSI, FW and GW as behavioral indicators to evaluate the effects of acupuncture on depressive-like symptoms. 


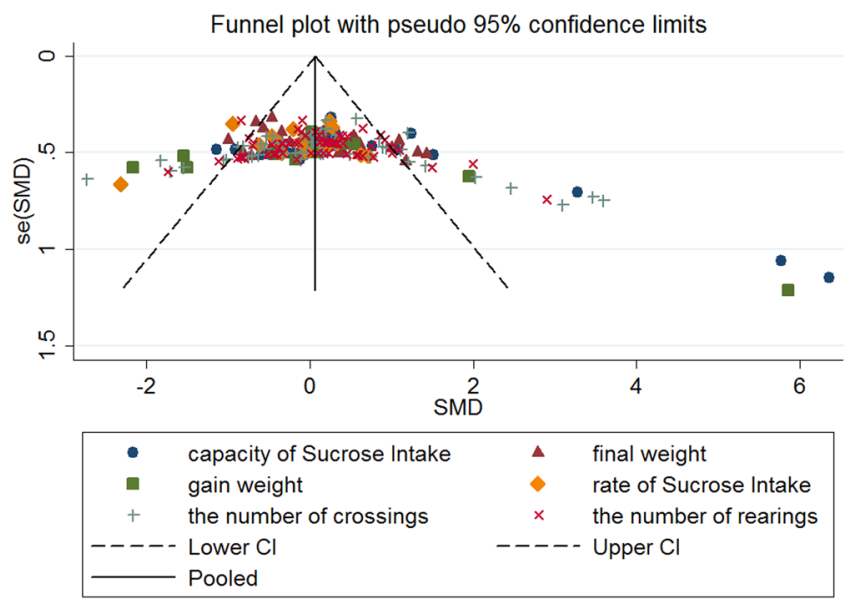

Figure 8. The funnel plot of the effects of acupuncture on behavioral changes in experimental depression.

Successful animal models of depression should meet the following conditions ${ }^{99}$ : (1) methods of modeling should have relations to pathogenesis of depression; (2) behavioral symptoms of models should be similar to clinical symptoms of depression; (3) the changes of pathophysiology in models should be similar to the changes of pathophysiology in depression; (4) antidepressant drugs are effective on behavioral symptoms of models; (5) behavioral symptoms of models should exist enough time for purpose of observing the effect of the treatment. A majority of studies in our analysis selected chronic stress models, which are most frequently used in depression experimental studies and considered to be with high validity, reliability and utility ${ }^{100}$, including CUMS, CUMS combined with solitary, CFSS ${ }^{11}, \mathrm{CRS}^{26}, \mathrm{LH}^{39}$, and PSA models ${ }^{95}$. Four studies made models on the basis of underlying pathogenesis of depression, included IA models ${ }^{22}$ (IA can simulate effects of the endogenous excitatory amino acids on the onset of depressive symptoms), L-AAA models (L-AAA can destroy astrocytes) ${ }^{64}$, EAAT1 antagonist models and EAAT2 antagonist models ${ }^{77}$ (EAAT1 and EAAT2 antagonist can inhibit functions of glutamate transporter). Results of these studies showed that the models were all successful. Nevertheless, it needs further researches to demonstrate which models have the highest validity, reliability and utility.

A Cochrane review has suggested that there is not enough evidence to demonstrate acupuncture is effective for depression and it has recommended future research should employ comparative designs and compare acupuncture with structured psychotherapies such as standard care ${ }^{101}$. Therefore, we compared acupuncture with western medicine, which can be considered as standard treatment in animal models at present. Current available anti-depressants are limited by lagged therapeutic time and undesirable side effects including acute nausea and headaches as well as chronic sexual dysfunction, weight gain, and diminished REM (rapid eyes movement) sleep ${ }^{2}$. We expected that acupuncture may have not less effect than anti-depressants on depression and have an advantage on less side effects ${ }^{5,102}$, which is helpful for patients to adhere to the treatment.

Mechanisms of acupuncture. The mechanisms of acupuncture on depression remain unclear. Main possible mechanisms of acupuncture include: (1) regulating the level of neurotransmitter such as monoamine neurotransmitter (5-HT, DA, NE ${ }^{11,71,84,93}$ ), Glu and GABA; (2) regulating neuroendocrine system (HPA axise ${ }^{88,93}$, HPT axise ${ }^{52}, \mathrm{MT}^{59}$ ); (3) regulating inflammatory cytokines such as IL-1 $\beta$, IL-2, IL-6, TNF- $\alpha$, NO, PGE2, iNOS, $\mathrm{COX}-2, \mathrm{NF}-\mathrm{kB}^{21,27,49}$; (4) promoting neuronal regeneration and neurotrophy through several signaling pathways (including BDNF, CREB, PKA, JNK, ERK, etc) ${ }^{15,19,25,32,37,46,89}$. In addition, acupuncture can modulate brain-gut peptide hormone (including GAS, NPY, CGRP, NT, etc) ${ }^{55,69}$, inhibit over-activation of RAS and suppress oxidative stress ${ }^{59,62}$. We consider that acupuncture treat depression by multi-targets through neuroendocrine-immune system and the future researches should enlarge the sample size and further clarify the pathophysiological effects of different acuopints or acupoint combinations.

Inspirations for further studies. As shown in Table S1, acupoints that were most frequently used (over 10 studies) include scalp acupoints (GV20, GV29) and body acupoints (LR3, PC6, LI4, SP6). We expected that acupoints may be a potential source of heterogeneity among these studies. Hence, subgroup analysis according to different stimulation acupoints (scalp acupoints, body acupoints, or scalp acupoints and body acupoints) based on NC and GW was conducted (Table 4). Nevertheless, results showed that different stimulation acupoints may not be the source of heterogeneity. Due to some unexpected influences of acupuncture parameters (such as the stimulation dose of acupuncture, different meridians, and so on) and small sample size of included studies, it is difficult to deny acupoints' contribution to the heterogeneity among the included studies.

It is also interesting to find that methods for selection of acupoints included on the basis of traditional Chinese medicine theory (49 studies), clinical experience (6 studies), preliminary clinical and/or experimental researches (19studies), summary of previous research reports (15 studies). This means that it doesn't have a standard theoretical guidance for selection of acupoints. We suggest that future studies should clarify the physiological effects of different acupoints or acupoint combinations and the differences in their effects on depression. 


\begin{tabular}{|c|c|}
\hline Research & Proposed mechanisms \\
\hline Bao $2014^{11}$ & Increased 5-HT in serum. \\
\hline Bao $2014 \#^{12}$ & Relieved impairment of hippocampal neurons. \\
\hline \multirow{2}{*}{ Chen $2011^{13}$} & Increased expression of $\mathrm{Bcl}-2$ in hippocampus. \\
\hline & Decreased expression of Bax in hippocampus. \\
\hline Cheng $2015^{14}$ & Regulated the expression of IL-6R in frontal cortex and hippocampus. \\
\hline \multirow{2}{*}{ Dai $2010^{15}$} & Down-regulated level of phospho-JNK in hippocampus. \\
\hline & Reduced hippocampal apoptotic rate. \\
\hline Deng $2013^{16}$ & Decreased expressions of IL-1 $\beta$, IL-6 in hippocampus and serum. \\
\hline Duan $2008^{18}$ & Increased p-CREB in hippocampus. \\
\hline \multirow{3}{*}{ Duan $2016^{19}$} & Increased 5-HT, Glu, GABA in hippocampus. \\
\hline & Increased levels of TrkB, BDNF, p-CREB,PKA and decreased CaMKII in the hippocampus. \\
\hline & $\begin{array}{l}\text { Up-regulated expression levels of PKA mRNA and TrkB mRNA and down-regulated expression levels of } \\
\text { CaMKII mRNA in the hippocampus. }\end{array}$ \\
\hline \multirow{4}{*}{ Duan $2016 \#^{20}$} & Increased 5-HT in hippocampus. \\
\hline & Increased 5-HIAA level. \\
\hline & Up-regulated levels of TPH and 5-HT1A. \\
\hline & Up-regulated expression levels of TPH mRNA and 5-HT1A mRNA. \\
\hline Fan $2013^{21}$ & Decreased IL-2,IL-6 and NO in serum. \\
\hline Fan $2016^{22}$ & Regulated levels of 5-HT, NE, BDNF and expression of Bax/Bcl-2. \\
\hline Fan $2016 \#^{23}$ & Down-regulated expressions of SNAP25, VAMP1, VAMP2, VAMP7 and syntaxin1. \\
\hline Fan $2016 * 24$ & Down-regulated expressions of SNAP25, VAMP1, VAMP2, VAMP7 and syntaxin1. \\
\hline Fu $2008^{25}$ & Up-regulated expressions of CREB, BDNF in hippocampus. \\
\hline \multirow{2}{*}{ Guo $2016^{26}$} & Decreased level of ROS in hippocampus. \\
\hline & Decreased protein expression levels of cytochrome C,caspase-3, and AIF in hippocampus \\
\hline Hu $2013^{27}$ & Decreased IL-1 $\beta, I L-6, T N F-\alpha$ in serum, hippocampus and frontal cortex. \\
\hline \multirow{2}{*}{ Hu $2014^{28}$} & Down-regulated expressions of RAGE, Activin A, CNTF R $\alpha$, EGFR, E-Selectin, and Resistin in hippocampus. \\
\hline & Decreased IL-1 $\beta$, IL-10 in hippocampus. \\
\hline Huang $2005^{29}$ & Decreased CORT,ACTH in serum. \\
\hline Ji $2013^{30}$ & Increased the GFAP and astrocyte in hippocampus. \\
\hline \multirow{5}{*}{ Jia $2005^{31}$} & Decreased IL-1, CRF in hypothalamus. \\
\hline & Decreased CRF in hypophysis. \\
\hline & Decreased ACTH, CORT in adrenal gland. \\
\hline & Down-regulated expressions of $\mathrm{CRF}_{1} \mathrm{mRNA}, \mathrm{POMC} \mathrm{mRNA}$ in hypophysis. \\
\hline & $\begin{array}{l}\text { Up-regulated expressions of GRmRNA in hippocampus, hypothalamus, hypophysis and MRmRNA, } \\
\text { 5-HT }{ }_{1 \mathrm{~A}} \mathrm{mRNA} \text { in hippocampus. }\end{array}$ \\
\hline \multirow{2}{*}{ Jiang $2007^{32}$} & Incresed cAMP in hippocampus. \\
\hline & Up-regulated expressions of CREB,BDNF in hippocampus. \\
\hline \multirow{2}{*}{ Jiang $2013^{33}$} & Repaired astrocytes in CA1, DG area in hippocampus. \\
\hline & Increased GFAP, GFAPmRNA in hippocampus. \\
\hline Jin $2015^{35}$ & $\begin{array}{l}\text { Down-regulated expression of NF-kB, COX-2, and COX-2 mRN in hippocampus and decreased PGE2 in } \\
\text { hippocampus. }\end{array}$ \\
\hline Jing $2016^{36}$ & Down-regulated the expression of PDE4A and PDE4D mRNA in hippocampus. \\
\hline Li $2007^{37}$ & Increased cAMP in hippocampus. \\
\hline \multirow{2}{*}{ Li $2008^{38}$} & Increased bcl-2 in hippocampus. \\
\hline & Reduced hippocampal apoptotic rate. \\
\hline Li $2011^{39}$ & Regulated the gene expression of Dnmt3L and MBP in hippocampus. \\
\hline Li $2011 \&^{40}$ & Increased $\beta$-EP in serum and $\mu$ receptors level in hypothalamus. \\
\hline Li $2014^{41}$ & Increased 5-HT and 5-HTT in hippocampus. \\
\hline Lin $2008^{42}$ & Increased BDNF in the hippocampal. Dncreased TGF- $\beta 1$ in serum. \\
\hline Liu $2008^{44}$ & Regulated GFAP,BDNF,GDNF in hippocampus. \\
\hline Liu $2009^{45}$ & Increased 5-HT,DA,NE in hippocampus. \\
\hline Liu $2012^{46}$ & Incresed AC transformation ratio,cAMP level, and PKA activity. \\
\hline \multirow{2}{*}{ Lu $2008^{47}$} & Reduced CORT in serum. \\
\hline & Increased expressions of PKA and PKC in hippocampus. \\
\hline Lu $2013^{48}$ & Regulated levels of ERK1/2,p-ERK1/2, CREB, p-CREB in the hippocampus. \\
\hline \multirow{2}{*}{ Lu $2016^{49}$} & Reduced levels of NO, PGE2, iNOS and COX-2 in the hippocampus and prefrontal cortex. \\
\hline & Inhibited the activation of NF-kB in the hippocampus and prefrontal cortex. \\
\hline
\end{tabular}




\begin{tabular}{|c|c|}
\hline Research & Proposed mechanisms \\
\hline Lu $2016 \#^{50}$ & $\begin{array}{l}\text { Increased the mRNA and protein expression of IL-1 } 13 \text {, IL-6, and TNF- } \alpha \text { in the hippocampus and prefrontal } \\
\text { cortex and cytokine concentrations in serum. }\end{array}$ \\
\hline Luo $2016^{51}$ & Regulated GS,EAAT1,EAAT2 of astrocyte cell in prefrontal cortex. \\
\hline Ma $2016^{52}$ & Upregulated expression of TRH and TRH mRNA in the hypothalamus. \\
\hline Mo $2014^{53}$ & Upregulated expression of TRH and TRH mRNA in the hypothalamus. \\
\hline \multirow{3}{*}{ Pan $2016^{54}$} & Regulated Glu, GABA in hippocampal \\
\hline & Increased DA,5-HT in serum. \\
\hline & Regulated expressions of GAD65, EATT3,GAT1, NR2B in hippocampal. \\
\hline Qin $2010^{55}$ & Increased NT in hypothalamus and ileum. \\
\hline Shao $2016^{56}$ & Downregulated expressions of NF-kB, NO, iNOS. \\
\hline Shi $2007^{57}$ & Reduced levels of CORT,ACTH,CRH. \\
\hline Shi $2015^{58}$ & Increased 5-HT in hippocampal and BDNF in serum levels,and decreased CORT in adrenal. \\
\hline \multirow{4}{*}{ Song $2014^{59}$} & Increased the production of MT. \\
\hline & Regulated ET, NO, ET-1 in serum. \\
\hline & $\begin{array}{l}\text { Regulated leves of renin Ang II (Angiotensin II), AT1R (Angiotensin II Type } 1 \text { Receptor), ACE (Angiotensin } \\
\text { converting enzyme). }\end{array}$ \\
\hline & Regulated MOD, SOD in serum. \\
\hline Song $2015^{60}$ & Regulated BDNF, bcl-2, CREB,and EPK signaling pathway. \\
\hline Song $2016^{61}$ & Reduced CORT,ACTH,CRH in serum. \\
\hline Song 2014\#" $\#^{62}$ & Down-regulated expression of AngII, AT1R and ACE I in the arterial tissue. \\
\hline Sun $2013^{64}$ & Promoted repair of astrocytes. \\
\hline Sun $2014^{65}$ & Down-regulated expression of p-JNK, c-jun, Caspase-3. \\
\hline Sun $2016^{66}$ & Decreased CORT, ACTH in serum. \\
\hline Tang $2013^{67}$ & Increased 5-HT,DA,NE in hippocampus. \\
\hline Tang $2014^{68}$ & Increased 5-HT,DA,NE in hippocampus and loubus fromatis. \\
\hline \multirow{2}{*}{ Teng $2013^{69}$} & Increased GAS and reduced NPY,CGRP in colonic mucosa,increased GAS,NPY,CGRP in hypothalamus. \\
\hline & Increased $\beta$-EP in hypothalamus and reduced $\beta$-EP in colonic mucosa. \\
\hline \multirow{2}{*}{ Wang $2009^{71}$} & Incresed expression of 5-HT2A receptor mRNA in hippocampus. \\
\hline & Reduced expression of 5-HT1A receptor mRNA in hippocampus. \\
\hline Wang $2010^{72}$ & Increased BDNF in hippocampus. \\
\hline Wang $2013^{73}$ & Regulated $\beta$-EP in serum and brain tissue. \\
\hline Wang $2016^{75}$ & Promoted repair of hippocampus $\mathrm{CA} 3$ region. \\
\hline \multirow{2}{*}{ Wu $2007^{76}$} & Repaired the lesion of multiple organs. \\
\hline & Promoted 5-HT synthesis. \\
\hline Xiao $2014^{77}$ & Improved expression of GS mRNA, EAAT1 mRNA,EAAT2 mRNA. \\
\hline Xiao $2014 \&^{78}$ & Improved the injury of hippocampal astrocytes. \\
\hline Xu $2016^{79}$ & Up-regulated level of TGF-3 $\beta$ in hippocampal and down-regulated expression of bFGF. \\
\hline Xu $2016 \#^{80}$ & Regulated levels of ERK1/2, p-ERK1/2, CREB, p-CREB and BDNF in the hippocampus. \\
\hline Yang 2013\# $\#^{82}$ & Regulated levels of TrkB and BDNF. \\
\hline Yu $2012^{84}$ & Increased 5-HT,DA,NE in brain. \\
\hline Yu $2016^{85}$ & Regulated levels of PKA,CREB in prefrontal cortex and IL-6,TNF- $\alpha$ in serum. \\
\hline \multirow{2}{*}{ Zhang $2008^{88}$} & Reduced CORT and CRF. \\
\hline & Improved expression of GR,NMDA,NR2B, PKA, CREB, Nestin in hippocampus. \\
\hline Zhang $2016^{89}$ & Regulated levels of ERK1/2,p-ERK1/2, CREB, p-CREB and BDNF in the hippocampus. \\
\hline \multirow{2}{*}{ Zhang\# $2016^{90}$} & Regulated Glu,GABA in hippocampus. \\
\hline & Down-regulated expressions of NMDA-receptor subunits NRl and NR2A in hippocampus. \\
\hline Zhang 2016\&:11 & Increased GFAP,GS,Glu-C4,Gln-C4,GABA-C2 in prefrontal cortex and hippocampus. \\
\hline Zhang $2016^{* 92}$ & Regulated BDNF, acH3K9, HDAC2 and PKA signaling pathway. \\
\hline \multirow{2}{*}{ Zhao $2005^{93}$} & Reduced CORT,ACTH in serum. \\
\hline & Increased 5-HT, NE in brain. \\
\hline Zheng $2013^{94}$ & Down-regulated expression of CRF in hypothalamus. \\
\hline
\end{tabular}

Table 3. Proposed mechanisms of acupuncture in behavior changes in depression models of studies included. Notes: $\beta$-EP, $\beta$-endorphin; -CREB, phosp-horylated cAMP response element binding protein; 5 -HT, 5-hydroxytryptamine; 5-HT1A: 5-hydroxyindoleacetic acid; 5-HTT, 5-hydroxytryptamine transporter; ACEI, Angiotensin converting enzyme I; acH3K9, acetylation of histone 3 lysine 9; ACTH, adrenocorticotropic hormone; AIF, apotposis inducing factor; AngII, angiotensin II; AT1R, angiotensin II Type 1 Receptor; Bax, Bcl-2 Assaciated X protein; Bcl-2, B-cell lymphoma 2; BDNF, brain derived neurotrophic factor; bFGF, basic fibroblast growth factor; CaMKII, calcium-calmodulin-dependent protein kinase II; cAMP, adenosine 3', 5'-cyclic monophos-phate; CGRP, calcitonin generelated peptide; CNTF R $\alpha$, ciliary neurotrophic factor 
receptor $\alpha$; CORT, corticoserone; COX-2, cyclo-oxygenase-2; CRF, corticotropin releasing factor; CRH, corticotropin releasing hormone; DA, Dopi Amine; Dnmt, DNA methytransferase; EAAT1, excitatory amino acid transporter 1; EAAT2, excitatory amino acid transporter 2; EGFR, epithelial growth factor receptor; ERK, extracellular signal-regulated kinase; GABA, $\gamma$-aminobutyric acid; GAS, Gastrin; GDNF, glial cell line-derived neurotrophic factor. GFAP, glial fibrillary acidic protein; Gln, glutamine; Glu, glutamate; GR, glucocorticoid receptor; GS, glutamine synthetase; HDAC2, histone deacetylase 2; HPA, hypothalamus-pituitary-adrenal; IL-1 $\beta$, Interleukin-1 $\beta$; IL-6, interleukin-6; IL-6r, Interleukin-6r; IL-10, Interleukin-10; iNOS, inducible Nitric Oxide Synchase; JNK, c-Jun N-terminal kinasesignal; MBP: myelin basic protein; MLT, motilin; MOD, Malondialdehyde; MT, melatonin; NE, norepinephrine; Nestin, neuroepithelial stem protein; NF-kB, nuclear factor kappa B; NMDA, N-methy-D-aspartate; NO, nitric oxide; NPY, neuropeptide-y; NR2B, NMDA receptor subunits 2B; NT, neurotensin; PDE4, phosphodiesterase 4; PGE2, prostaglandin E2; PKA, protein kinase A; PKC, protein kinase C; RAGE, receptor for advanced glycation end-products; RAS, renin-angiotensin system; ROS, reactive oxygen species; SNAP, synaptic soluble Nethylmaleimide-sensitive factor attachment receptor; SOD, Superoxide dismutase; SS, somatostatin; TGF-3 $\beta$, transforming growth factor beta 3; TNF- $\alpha$, tumor necrosis factor- $\alpha$; TPH, tryptophan hydroxylase; TRH, Thyrotropin releasing hormone; TrkB, tropomyosin receptor kinase B; VAMP, vesicle-associated membrane protein.

\begin{tabular}{|l|l|l|}
\hline Sensitivity analysis & Removed studies & Changes of $\mathbf{I}^{\mathbf{2}} \mathbf{( P )}$ \\
\hline NR & Jing $2016^{36}$, Fan2013 ${ }^{21}$ & $44 \%(0.0002) \rightarrow 29 \%(0.02)$ \\
\hline CSI & Zhang2008 ${ }^{88}$, Fan2013 ${ }^{21}$, Yang $2013^{81}$ & $73 \%(0.04) \rightarrow 32 \%(0.06)$ \\
\hline RSI & Sun2016 ${ }^{66}$ & $43 \%(0.04) \rightarrow 14 \%(0.30)$ \\
\hline Subgroup analysis & Factors & $\mathrm{I}^{2}$ in Subgroups \\
\hline NC & different types of acupuncture & EA: 69\%, MA:76\% \\
\hline & different stimulation acupoints & SA:55\%, BA:74\%, SA+BA:78\% \\
\hline & different intervention time & SAME:74\%, NOT SAME:66\% \\
\hline & different duration of treatment & More than 21d:72\%, Less than 14d:41\% \\
\hline GW & different stimulation acupoints & SA:89\%, BA:86\%, SA+BA:73\% \\
\hline & different intervention time & SAME:96\%, NOT SAME:0\% \\
\hline
\end{tabular}

Table 4. Details of sensitivity analysis and subgroup analysis. Notes: CSI:capacity of sucrose intake; EA:electroacupuncture; GW:gain weight; MA: manual acupuncture; NC: the number of crossings; NOT SAME: intervention after or in the process of modeling. NR: the number of rearings; RSI:rate of sucrose intake; SA:scalp acuponits; BA:body acupoints; SAME:intervention and modeling at the same time.

Methodological quality of the included studies was generally low and only one of them got 6 points and two got 7 in our assessment. Randomization, blinding and sample-size estimation serve as the core standards of rigorous study design. In our analysis, only two studies did not describe randomization, but none of the studies described the sample size calculation, allocation concealment, blinded assessment of outcome. Blinding should also be utilized in intervention process. Research shows that the visual impact of needling is a potential factor that leads to the placebo effect of acupuncture ${ }^{103}$. Therefore, we suggest that animals should be prevented from seeing themselves or their companions being needled in intervention process. And more attention should be paid to the methodological quality in future studies.

In the sensitivity analysis, we considered that Jing $2016^{36}$, Fan $2013^{21}$ were the major sources of heterogeneity in NR, Zhang $2008^{88}$, Fan $2013^{21}$,Yang $2013^{81}$ were the major sources of heterogeneity in CSI and Sun $2016^{66}$ were the major sources of heterogeneity in RSI. We found that quality of Zhang $2008^{88}$, Fan $2013^{21}$ and Yang $2013^{81}$ are considerable poor (one got 2 points and the other two got 3 points). We believe that avoiding publication bias and improving methodological quality of studies play an important role in reducing the heterogeneity in studies.

In the subgroup analysis, we did not find the exact factor that accounts for the heterogeneity among studies pooled in the meta-analysis of NC or GW. We also developed the meta regression and the result showed that factors of acupuncture treatment did not account for the heterogeneity in studies (Figs S11 and S12). More possible factors that can influence outcomes, such as experimental models, should be considered in later researches to interpret the heterogeneity among studies.

Methodological interpretations. There were some methodological limitations in our study. First of all, we failed to meta-analyze all data because of insufficient data in several primary studies included. It is unclear whether the result would be changed or not when insufficient data were added in the analysis. Secondly, our study did not include data in other languages except for Chinese and English, which may result in certain degree of selective bias. Third, publication bias existed demonstrated by the asymmetry of the funnel plot and statistical analysis with Egger's test. Some non-positive studies have been missed inevitably, as negative findings are less likely to be published. All the studies were published by Chinese authors, and only 1/9 of them were published in English journals. We cannot rule out the possibility that cultural difference is an underlying cause of publication bias. The above-mentioned methodological limitations suggested that the results should be interpreted with caution. 


\section{Conclusions}

Acupuncture has not less effect than western medicine on behavior indicators including NC, NR, FW, GW, and RSI. These indicators can be utilized to evaluate acupuncture effectiveness in experimental depressive disorder. Nevertheless, the results of this meta-analysis need to be interpreted with caution and high-quality researches are urgently needed. Future studies should pay more attention to the methodological quality, especially in sample size calculation, allocation concealment, blinding in intervention and assessment. Additionly, we suggest that future studies should further clarify the physiological effects of different acupoints or acupoint combinations and the differences in their effects on depression.

\section{Methods}

Search strategy. We searched the following databases from their inception up to January 13, 2017: PubMed, EMBASE, Cochrane Central Register of controlled trials(CENTRAL), China National Knowledge Infrastructure (CNKI), Wanfang data Information Site, and VIP information database. The search terms included '电针, “针刺, ‘针炎, ‘抑郁, ‘抑郁症, ‘郁证, ‘郁病’ in Chinese and 'electroacupuncture, ' acupuncture’, 'depression', 'depressive' in English. Besides, we also scanned the references of all the eligible studies carefully to identify further relevant publications.

Eligibilit. Inclusion Criteria: (1) The objects are depressive models rat in both experimental and control group; (2) Interventions in experimental groups are acupuncture/electroacupuncture and in control groups are western medicine; (3) Outcome indicators should include at least one of the following indicators: number of crossings (NC) or number of rearings (NR) in open field test(OFT), capacity of sucrose intake (CSI) or rate of sucrose intake (RSI) in sucrose intake test (SI), final weight (FW) or gain weight (GW).

Exclusion criteria: (1) Experimental models combined depression with other diseases or disorders; (2) The western medicine control group is not set; (3) None of the indicators mentioned in inclusion criteria is reported; (4) Duplicate publications.

Data extraction. We extracted the key contents of the studies in reference to the ARRIVE guideline ${ }^{104}$, and the following details were extracted: (1) publication year and the first author's name; (2) animal species, sex, weight; (3) the randomization and blinding; (4) interventions and modeling; (5) outcome indicators. When studies set up two or more acupuncture groups, the group which had greater effects on the outcome indicators was extracted. All continuous data of outcome indicators which was presented as mean \pm standard deviation (SD) were extracted if reported. Missing data or further information was sought from the primary authors via e-mail if necessary.

Quality assessment. We evaluated the methodological quality of the included studies by applying the list of Collaborative Approach to Meta-Analysis and Review of Animal Data from Experimental Stroke (CAMARADES) ${ }^{105}$ which is modified on the basis of the characteristics of acupuncture treatment in depression researches when assessing the quality of studies, the items included: (1) sample size calculation; (2) randomization to treatment group; (3) allocation concealment; (4) blinded assessment of outcome; (5) correctness of methods of modeling; (6) avoidance of anesthetics with resistance to depressive; (7) describing control of temperature; (8) compliance with animal welfare regulations; (9) publication in a peer-reviewed journal; (10) declared any potential conflict of interest. For the calculation of an aggregate quality score, each item was attributed one point. Two authors independently extracted data and assessed study quality. Disagreements were solved after discussion over the details of the studies.

Statistical Analysis. Meta-analysis and subgroup-analysis were performed by RevMan V.5.3, and analysis of publication bias and Meta-regression were conducted with STATA/SE 12.0. We considered all behavioral indicators as continuous data, and then an estimate of the combined effect sizes utilizing standard mean difference (SMD) with the given random effects model. We used the random model rather than fixed since heterogeneity between multi-studies has to be taken into account. Publication bias was assessed with a funnel plot and Egger's test. To assess heterogeneity, the $\mathrm{I}^{2}$ statistic was used. To clarify the impact of factors potentially modifying the outcome measures, we also conducted sensitivity analysis and subgroup analysis according to the following variables: different types of acupuncture, different stimulation acupoints, different intervention time, different duration of treatment. The difference between groups was assessed by partitioning heterogeneity and using the $\chi^{2}$ distribution with $\mathrm{n}-1$ degrees of freedom $(\mathrm{df})$, where $\mathrm{n}$ indicate the number of groups. Probability value $\mathrm{p}<0.05$ was considered significant.

Data Availability. All data generated or analysed during this study are included in this published article (and its Supplementary Information files).

\section{References}

1. MacPherson, H. et al. Acupuncture and Counselling for Depression in Primary Care: A Randomised Controlled Trial. Plos Med. 10, e1001518 (2013)

2. Gerhard, D. M., Wohleb, E. S. \& Duman, R. S. Emerging Treatment Mechanisms for Depression: Focus On Glutamate and Synaptic Plasticity. Drug Discov Today. 21, 454-464 (2016).

3. Xu, J. et al. Effects of Electroacupuncture on Chronic Unpredictable Mild Stress Rats Depression-Like Behavior and Expression of p-ERK/ERK and p-P38/P38. Evidence-based complementary and alternative medicine: eCAM. 2015, 650729 (2015).

4. Qu, S. S. et al. A 6-Week Randomized Controlled Trial with 4-Week Follow-Up of Acupuncture Combined with Paroxetine in Patients with Major Depressive Disorder. J Psychiatr Res. 47, 726-732 (2013). 
5. Zhang, Z. J., Chen, H. Y., Yip, K. C., Ng, R. \& Wong, V. T. The Effectiveness and Safety of Acupuncture Therapy in Depressive Disorders: Systematic Review and Meta-Analysis. J Affect Disorders. 124, 9-21 (2010).

6. Berton, O., Hahn, C. G. \& Thase, M. E. Are we Getting Closer to Valid Translational Models for Major Depression? Science (New York, N.Y.). 338, 75-79 (2012).

7. Yang, J. et al. Enhanced Antidepressant-Like Effects of Electroacupuncture Combined with Citalopram in a Rat Model of Depression. Evidence-based complementary and alternative medicine: eCAM. 2013, 107380 (2013).

8. Yu, J. et al. Electroacupuncture Combined with Clomipramine Enhances Antidepressant Effect in Rodents. Neuroscilett. 421, 5-9 (2007).

9. Stanford, S. C. The Open Field Test: Reinventing the Wheel. Journal of psychopharmacology (Oxford, England). 21, 134-135 (2007).

10. Lin, Y. H. et al. Effect of Chronic Unpredictable Mild Stress On Brain-Pancreas Relative Protein in Rat Brain and Pancreas. Behav Brain Res. 165, 63-71 (2005).

11. Bao, W. Y. et al. Effects of Electroacupuncture on Depressive-Like Behavior and Serum 5-HT in the Chronic Forced Swim Stress Model Rats. Information on Traditional Chinese Medicine. 31, 44-47 (2014).

12. Bao, W. Y. et al. Effect of Electroacupuncture Intervention on Learning-Memory Ability and Injured Hippocampal Neurons in Depression Rats. Acupuncture. Research. 39, 136-141 (2014).

13. Chen, L. \& Wang, L. L. Effects of 'Tong Du Tiao Shen' Acupuncture On Bax and Bcl-2 in Depression Rats. Acta Chinese Medicine and Pharmacology. 39, 85-88 (2011)

14. Cheng, H. Effect of Electric Acupuncture and Drug Combination On IL-6R Expression in the Related Encephalic Region of Depression rats(MD. Thesis, in Chinese). Available at: http://d.wanfangdata.com.cn/Thesis/D744770 (Accessed 13th Jan 2017) (2015).

15. Dai, W., Li, W. D., Lu, J. \& Tu, Y. Effect of Electroacupuncture on Hippocampal Apoptosis and JNK Signal Pathway in Chronic Stress Depression Rats. Acupuncture. Research. 35, 330-334 (2010).

16. Deng, X. F. Influence on the Expression of IL-1 $\beta$, IL-6 in Hypothalamus and Serum of Rats with Chronic Unpredictable Mild Stress-Induced Depression by Different Electro-Acupuncture Treatment (MD. Thesis, in Chinese). Available at: http://www.cnki. net/KCMS/detail/detail.aspx?FileName=1013206413.nh\&dbname=CMFD201302 (Accessed 5th Aug 2015) (2013).

17. Ding, N., Li, R. \& Tian, H. H. The Effects and Difference of Acupuncture and Moxibustion on the Behavior in Depression Rats of CUMS. Guiding Journal of Traditional Chinese Medicine and Pharmacy. 22, 49-51 (2016).

18. Duan, D. M., Tu, Y. \& Chen, L. Effects of Electroacupuncture at Different Acupoint Groups On Behavior Activity and p-CREB Expression in Hippocampus in the Rat of Depression. Chinese Acupuncture \& Moxibustion. 28, 369-373 (2008).

19. Duan, D. M., Tu, Y., Liu, P. \& Jiao, S. Antidepressant Effect of Electroacupuncture Regulates Signal Targeting in the Brain and Increases Brain-Derived Neurotrophic Factor Levels. Neural Regen Res. 11, 1595-1602 (2016).

20. Duan, D. M., Tu, Y., Yang, X. Y. \& Liu, P. Electroacupuncture Restores 5-HT System Deficit in Chronic Mild Stress-Induced Depressed Rats. Evid-Based Compl Alt. 2016, 1-9 (2016).

21. Fan, M. C. Effects of Acupuncture On the Change of Behavior and Level of Cytokines in Depression Model Rats (MD. Thesis, in Chinese). Available at: http://www.cnki.net/KCMS/detail/detail.aspx?FileName=1013229286.nh\&DbName=CMFD2013 (Accessed 5th Aug 2015) (2013).

22. Fan, L. et al. Effects of Acupuncture On the Levels of 5-HT, NE, BDNF and Expression of Neuronal Apoptosis Related Genes in Hippocampus of the Depression Rats. China Journal of Traditional Chinese Medicine and Pharmacy. 31, 3204-3207 (2016).

23. Fan, L. et al. Effect of Acupuncture On the Release of Presynaptic Glutamate Mediated by SNARE Protein in Hippocampus of Rats with Depression. Chinese. Journal of Gerontology. 36, 3123-3126 (2016).

24. Fan, L. et al. Soluble N-Ethylmaleimide-Sensitive Factor Attachment Receptor (SNARE) Protein Involved in the Remission of Depression by Acupuncture in Rats. Journal of Acupuncture and Meridian Studies. 9, 242-249 (2016).

25. Fu, W. B. A Randomized Controlled Study on Acupuncture Treatment of Regulating Liver and Spirit for Depression and the Postreceptor Signal Transduction Mechanism(PhD. Thesis, in Chinese). Available at: http://d.g.wanfangdata.com.cn/Thesis_ D072969.aspx (Accessed 5th Aug 2015) (2008).

26. Guo, Y. Effecs of Acupuncture On Regulating the Content of Reactive Oxygen and the Inhibition of Mitochondrial Apoptosis pathway(MD. Thesis, in Chinese). Available at: http://www.cnki.net/KCMS/detail/detail.aspx?FileName=1016069050. nh\&DbName $=$ CMFD2016 (Accessed 13th Jan 2017) (2016).

27. Hu, L. Experimental Study of Influences of Acupuncture On Inflammatory Cytokines of Serum and Brain in Chronic Stress Depression models(MD. Thesis, in Chinese). Available at: http://www.cnki.net/KCMS/detail/detail.aspx?FileName=1013204417. nh\&DbName $=$ CMFD2013 (Accessed 5th Aug 2015) (2013).

28. Hu, C. Preliminary Study of Associated Protein of Hippocampal Neurons for Antidepressant Effects of Acupuncture with Biotin Label-Based antibody(PhD. Thesis, in Chinese). Available at: http://www.cnki.net/KCMS/detail/detail. aspx?FileName $=1014242159$. nh $\&$ DbName $=$ CMFD2014 (Accessed 5th Aug 2015) (2014).

29. Huang, L. Experimental Study of Effects on HPA Axis of Chronic Stress Rat Model of Depression by Acupuncture the Right Neiguan and Taichong(MD. Thesis, in Chinese). Available at: http://d.g.wanfangdata.com.cn/Thesis_Y764072.aspx (Accessed 5th Aug 2015) (2005)

30. Ji, Q. et al. Influence of Music Electro-Acupuncture and Pulsed Electro-Acupuncture to the Hippocampus Astrocytes of Chronic Unpredictable Mild Stress Depression Model Rats. China Journal of Traditional Chinese Medicine and Pharmacy. 28, 648-651 (2013).

31. Jia, B. H. Experimental Study of Effect of Electroacupuncture On HPA Axis in Chronic Stress Depression Rats(PhD. Thesis, in Chinese). Available at: http://www.cnki.net/KCMS/detail/detail.aspx?FileName=2005077051.nh\&DbName=CDFD2005 (Accessed 5th Aug 2015) (2005)

32. Jiang, J. Study on the Effect of the Combination of Acupuncture and Antidepressant on the Mechanisms of the Hippocampus Neurons Signal Transduction of Depressed Model Rats and Clinical Research(PhD. Thesis, in Chinese). Available at: http://www. cnki.net/KCMS/detail/detail.aspx?FileName=2007223617.nh\&DbName=CDFD2013 (Accessed 5th Aug 2015) (2007).

33. Jiang, S. The Mechanism On the Repair of Electro-Acupuncture On Hippocampal Astrocytes in Chronic Stress Induced Depression Modle rats(PhD. Thesis, in Chinese). Available at: http://www.cnki.net/KCMS/detail/detail.aspx?FileName=1013226451. nh\&DbName $=$ CDD2013 (Accessed 5th Aug 2015) (2013).

34. Jiao, S., Yang, X., Wang, Y. \& Tu, Y. The Effects of Electroacupuncture On Open-Field Behavior and Recognitive Function of the Chronic Stress Model Rats. Chinese Journal of Behavioral Medical Science. 17, 114-116 (2008).

35. Jin, S. Y. Effects of Acupuncture On the NF-kB,COX-2,PGE2 in a Chronic Stress Rat Model of depression(MD. Thesis, in Chinese). Available at: http://www.cnki.net/KCMS/detail/detail.aspx?FileName=1015386224.nh\&dbname=CMFD201502 (Accessed 13th Jan 2017) (2015)

36. Jing, Q. K. et al. Effect of Electroacupuncture On Expression of Phosphodiesterase 4 and Subtypes in Hippocampus in Rats with Chronic Stress Depression. Chin J Rehabil. Theory Pract. 22, 1011-1014 (2016).

37. Li, W. D. et al. Effect of Electroacupuncture On Behavior of Chronic Stress Depression Rats and the Level of cAMP in Hippocampus. Chin J of Behavioral. Med Sci. 16, 1060-1063 (2007).

38. Li, W. D. et al. Effects of Electroacupuncture and Fluoxetine On Behavior and Apoptosis of Hippocampal Cells in Chronic Stress Rat. Chinese Journal of Psychiatry. 41, 237-240 (2008) 
39. Li, B. Changes of Behavior and Epigenetic Modification During Treatment of Electro-acupuncture and Antidepressant drugs $(\mathrm{PhD}$. Thesis, in Chinese). Available at: http://www.cnki.net/KCMS/detail/detail.aspx?FileName=1014446849.nh\&DbName=CDFD2015 (Accessed 5th Aug 2015) (2011).

40. Li, J. B., Wang, X. J., Wang, L. L., Lu, J. H. \& Chen, M. The Influence of $\beta$-Endorphin and its Receptor On Electro-acupuncture's Quick Effects On Anti-depression. Journal of Nanjing University of Traditional Chinese Medicine. 27, 441-444 (2011).

41. Li, Y. J. et al. Effect on the Expression of 5-HT,5-HTT in Hippocampus of Chronic Unpredictable Mild Stress-Induced Depressive Rats by Electro-Acupuncture Pretreatment. Journal of Clinical Acupuncture and Moxibustion. 30, 57-60 (2014).

42. Lin, X. J. Experimental Study On the Effect of Music Electrical Acupuncture to the Neurotrophin of the Depressing Rat model(MD. Thesis, in Chinese). Available at: http://www.cnki.net/KCMS/detail/detail.aspx?FileName $=2008100815$. nh\&DbName $=$ CMFD2008 (Accessed 5th Aug 2015) (2008).

43. Liu, Q. et al. Effect of Electroacupuncture on Behavioral Changes of Chronic Unpredictable Stress-Induced Depression Model Rats. Shanghai Journal of Acupuncture and Moxibustion. 24, 41-43 (2005).

44. Liu, Q. Changes of Adult Hippocampal Neurogenesis During Treatment of Electroacupuncture with Depression Model rats $(\mathrm{PhD}$. Thesis, in Chinese). Available at: http://www.cnki.net/KCMS/detail/detail.aspx?FileName=2009019626.nh\&DbName=CDFD2009 (Accessed 5th Aug 2015) (2008).

45. Liu, J. J., Tian, Y. E., Chen, L. \& Li, M. Influence of Ethology in Chronicity Stress Depression Model Rat and Hippocampus Major Monamine Neurohormone Trans-Mitter Treated by Electroacupuncture. Contemporary. Medicine. 15, 35-37 (2009).

46. Liu, J. et al. Role of AC-cAMP-PKA Cascade in Antidepressant Action of Electroacupuncture Treatment in Rats. Evid-Based Compl Alt. 2012, 1-7 (2012).

47. Lu, F. et al. Effects of Electroacupuncture On Behavior, Plasma COR and Expressions of PKA and PKC in Hippocampus of the Depression Model Rat. Chinese Acupuncture \& Moxibustion. 28, 214-218 (2008).

48. Lu, J. et al. Acupuncture Activates ERK-CREB Pathway in Rats Exposed to Chronic Unpredictable Mild Stress. Evid Based Complement Alternat Med. 2013, 469765 (2013).

49. Lu, J. et al. Acupuncture Ameliorates Inflammatory Response in a Chronic Unpredictable Stress Rat Model of Depression. Brain Res Bull. 128, 106-112 (2016).

50. Lu, J., Shao, R. H., Hu, L., Tu, Y. \& Guo, J. Y. Potential Antiinflammatory Effects of Acupuncture in a Chronic Stress Model of Depression in Rats. Neurosci Lett. 618, 31-38 (2016).

51. Luo, D. et al. Effect of Electric Acupuncture On GS, EAAT1, EAAT2 of Astrocyte Cell in CUMS Rats' Prefrontal Cortex. Chinese. Journal of Gerontology. 36, 4143-4146 (2016).

52. Ma, R. et al. Effect of Two Kinds of Electro Acupuncture on the Expression of TRH in Hypothalamus of Rats with Chronic Stress Depression Model. Progress in Modern. Biomedicine. 16, 4242-4246 (2016).

53. MO, Y. P. et al. Influence On the Expression of TRH in Hypothalamus of Rats with Chronic Unpredictable Mild Stress -Induced Depression by Different Electro-acupuncture Treatment (In Chinese). Acta Chinese Medicine and Pharmacology. 42, $71-75$ (2014).

54. Pan, Q. J. Effect of Acupuncture On Behavior and Mechanism of CUMS Depression Rat model(PhD. Thesis, in Chinese). Available at: http://www.cnki.net/KCMS/detail/detail.aspx?FileName=1016208427.nh\&DbName=CDFD2016 (Accessed 13th Jan 2017) (2016).

55. Qin, L. N., Pei, Q. H. \& Tang, Q. S. Influences of Electro-Acupuncture On Body Weight, Food Intake and NT of Chronic Stress Model Rat. Beijing. Journal of Traditional Chinese Medicine. 29, 62-64 (2010).

56. Shao, R. H. Effects of Acupuncture On the NF-kB,iNOS,NO in a Chronic Stress Rat Model of depression(MD. Thesis, in Chinese). Available at: http://www.cnki.net/KCMS/detail/detail.aspx?FileName=1016069203.nh\&DbName=CMFD2016 (Accessed 13th Jan 2017) (2016)

57. Shi, R. Y. et al. Effect of Electroacupuncture at Baihui and Yintang on Body Weight and HPA Axis of Rat Depression Model. Journal of Clinical Acupuncture and Moxibustion. 23, 50-53 (2007).

58. Shi, R. Y. et al. Effect of Acupuncture Stimulation of Different Acupoint Groups on Levels of Stress Hormones and Serum BrainDerived Neurotrophic Factor in Depression Rats. Acupuncture. Research. 40, 444-448 (2015).

59. Song, H. T. Influences of Chronic Stress On Renin-Angiotensin System and Vascular Endothelial and the Effects of electroacupuncture(PhD. Thesis, in Chinese). Available at: http://www.cnki.net/KCMS/detail/detail.aspx?FileName=1014242683. nh\&DbName $=$ CDFD2014 (Accessed 5th Aug 2015) (2014).

60. Song, Y. Z. Effects of Acupuncture On ERK Signal Pathway in CUMS Model Rats (PhD. Thesis, in Chinese). Available at: http:// www.cnki.net/KCMS/detail/detail.aspx?FileName $=1015386372 . n h \& D b N a m e=C D F D 2015$ (Accessed 13th Jan 2017) (2015).

61. Song, X. J., Li, R., Ding, N. \& Tian, H. H. Influences and Differences of Tongdu Tiaoshen Method and Shugan Jieyu Method on the Changes of Behavior-and-Hypothalamus Pituitary Adrenal Axis in the Depressive Model Rats. Journal of Clinical Acupuncture and Moxibustion. 32, 64-68 (2016).

62. Song, H. T. et al. Alteration of Angii, AT1R and ACEI in Aorta Tissue with Chronicity Stress Depression Model Rats and Effect of Electro-Acupuncture. Chinese Journal of Integrative Medicine on Cardio/Cerebrovascular Disease. 12, 337-339 (2014).

63. Sun, H. \& Zhang, Y. Z. Effect of Acupuncture and Moxibustion at Baihui and Zusanli On Behavior of Mouse and Rat Depression Model. Journal of Clinical Acupuncture and Moxibustion. 19, 47-49 (2003).

64. Sun, J. et al. The Effect of Electroacupuncture On Depressive Rats's Behavior Induced by Intracerebroventricular Injection of L-AAA (In Chinese). Chinese journal of gerontology. 33, 1820-1822 (2013).

65. Sun, L. et al. Effects of Acupuncture and Electroacupuncture (EA) On Hippocampal p-JNK, C-Jun and Caspase-3 Expression in Rats with Chronic Stress Depression. Journal of Beijing University of Traditional Chinese Medicine. 37, 820-824 (2014).

66. Sun, D. L., Li, X. \& Liu, Q. H. The Influence of Electroacupuncture Combined with Fluoxetine for Behavioristics and HypothalamicPituitary-Adrenal Axis in Rats with Depressive Model. Journal of Psychiatry. 29, 4-8 (2016).

67. Tang, Y. S. et al. Regulative Effects of Music Electroacupuncture On Expressions of Hippocampus Monoamine Neurotransmitters in Rats with Chronic Stress Depression. Journal of Beijing University of Traditional Chinese Medicine. 36, 263-267 (2013).

68. Tang, Y. S. et al. Influence of Music Electro-Acupuncture and Pulsed Electro-Acupuncture on the Different Encephalic Regions of Monoamine Neurotransmitter Chronic Unpredictable Mild Stress Depression Model Rats. Journal of Clinical Acupuncture and Moxibustion. 30, 52-55 (2014).

69. Teng, J. Y. Cinical Observation and Experimental Study of Different Types of Electro- Acupuncture for Depression with Somatic Symptoms of Gastrointestinal Tract(PhD. Thesis, in. Chinese). Available at: http://www.cnki.net/KCMS/detail/detail. aspx?FileName $=1013205634$. nh $\&$ DbName $=$ CDFD2013 (Accessed 5th Aug 2015) (2013).

70. Wang, Y. J., Zhang, Y., Tian, Y. P., Hou, C. Y. \& Zhao, X. Effect of Acupuncture Combined with Medicine On Behavior of Chronic Stressed Depression Rat. Traditional Chinese Medical Research. 21, 12-15 (2008).

71. Wang, X. J. The Rapid-Onset of Antidepressant of Central 5-HT Receptor Mechanism and Clinic Study in Acupuncture Combining Medicine(PhD. Thesis, in Chinese). Available at: http://www.cnki.net/KCMS/detail/detail.aspx?FileName=2009251915. nh\&DbName $=$ CDFD2010 (Accessed 5th Aug 2015).

72. Wang, S. E., Hu, S. Y. \& Zhang, C. H. The Effects of Electro-Acupuncture On Behavior and the Level of BDNF in the Hippocampus of Rats with Chronic Stress Depression. Chinese Journal of Physical Medicine and Rehabilitation. 32, 419-423 (2010).

73. Wang, X. J., Wang, L. L., Lu, J. H., Xu, L. \& Lin, Y. H. Different Influence On Behavioral Performance and Beta-Endorphin Levels of Depression Model Rats Treated with Different Frequencies of Electro-Acupuncture. China Journal of Traditional Chinese Medicine and Pharmacy. 28, 2873-2876 (2013). 
74. Wang, J., Li, X. B., Wu, J. Y. \& He, W. B. The Praxiology Effect of Applying the Acupuncture Method of Unblocking Du Meridian to Regulate Shen On Depression Model Rats. Journal of Shanxi College of Traditional Chinese Medicine. 15(14-15), 18 (2014).

75. Wang, L. et al. Effect of Eletroacupuncture Intervention on Behavior Changes and Hippocampus CA3 Region in Depression Rats. Journal of Clinical Acupuncture and Moxibustion. 32, 66-69 (2016).

76. Wu, A. Q. et al. Effect and its Mechanism of Electroacupuncture at Zu San Li Point On Rats with Chronic Unpredictable Stress. Chinese. Journal of Anatomy. 30, 538-541 (2007).

77. Xiao, Y. The Research Based On Glutamate Impact of Astrocytes in Rat After. Experimental Depression of the acupuncture(PhD. Thesis, in Chinese). Available at: http://www.cnki.net/KCMS/detail/detail.aspx?FileName=1014359112.nh\&DbName=CDFD2015 (Accessed 5th Aug 2015) (2014).

78. Xiao, Y., Jiang, S., Fu, W. B. \& Pan, H. F. Effects of Electro-Acupuncture On Chronic Stress Which Induced Depression Rats' Hippocampal Structure and Function of AST Under TEM. China Journal of Traditional Chinese Medicine and Pharmacy. 29, $1682-1686(2014)$

79. Xu, M. M. et al. Effect of Eletroacupuncture Intervention On Behavior Changes and Levels of Hippocampal Transforming Growth Factor Beta 3 and Basic Fibroblast Growth Factor Proteins in Depression Rats. Acupuncture. Research. 41, 138-143 (2016).

80. Xu, M. M. Influence of Acupuncture in Regulating ERK1/2-CREB-BDNF Signal Pathway in Prefrontal Cortex of Depression Model rats(MD. Thesis, in Chinese). Available at: http://www.cnki.net/KCMS/detail/detail.aspx?FileName=1016069202. nh\&DbName $=$ CMFD2016 (Accessed 13th Jan 2017) (2016).

81. Yang, D. \& Zhai, S. Q. Effects of Electroacupuncture of Zusanli Acupoint on Behavior Changes Rats with of Chronic Stress Depressive Disorder. Acta Chinese Medicine and Pharmacology. 41, 6-8 (2013).

82. Yang, J. et al. Enhanced Antidepressant-Like Effects of Electroacupuncture Combined with Citalopram in a Rat Model of Depression. Evid Based Complement Alternat Med. 2013, 1-12 (2013).

83. Yu, J. et al. Effect of Electroacupuncture On Behavioral Activities of Depression Rats. Acupuncture. Research. 31, 31-34 (2006).

84. Yu, R. F. Influences On the Expression of 5-HT,DA, NE of Chronic Unpredictable. Stress Animal Models in Different Brain Regions of electro-acupuncture(MD. Thesis, in Chinese). Available at: http://www.cnki.net/KCMS/detail/detail. aspx?FileName $=1012363022 . n h \& D b N a m e=C M F D 2012$ (Accessed 5th Aug 2015) (2012).

85. Yu, M. Influence of Acupuncture in Regulating cAMP-PKA-CREB Signal Pathway in Prefrontal Cortex of Depression Model rats(MD. Thesis, in Chinese). Available at: http://www.cnki.net/KCMS/detail/detail.aspx?FileName=1016069178. nh\&DbName $=$ CMFD2016 (Accessed 13th Jan 2017) (2016).

86. Yu, J., Li, X. Y., Cao, X. D. \& Wu, G. C. Sucrose Preference is Restored by Electro-Acupuncture Combined with Chlorimipramine in the Depression-Model Rats. Acupuncture \&amp; electro-therapeutics research. 31, 223-232 (2006).

87. Zhang, J. B., Wang, L. L., Lv, M., Liu, L. Y. \& Li, D. Effect of Acupuncture in Inhibiting Behavior of Rats with Depression. Journal of Nanjing University of Traditional Chinese Medcine. 21, 36-39 (2005).

88. Zhang, M. Influences of Electro-Acupuncture and Chinese Herb of NY-3 On Pathway of HPA-Glu-NMDA in Chronic Stress models(PhD. Thesis, in Chinese). Available at: http://d.g.wanfangdata.com.cn/Thesis_Y1284203.aspx (Accessed 5th Aug 2015) (2008).

89. Zhang, X. H. et al. Antidepressant-Like Effects of Acupuncture Involved the ERK Signaling Pathway in Rats. BMC Complement Altern Med. 16, 380 (2016)

90. Zhang, L. H. Study On the Effects of Excitement and Inhibition of Depression Model Rats Hippocampal Glu/GABA by Acupuncture therapy(MD. Thesis, in Chinese). Available at: http://www.cnki.net/KCMS/detail/detail.aspx?FileName=1016185399. nh\&DbName $=$ CMFD2016 (Accessed 13th Jan 2017) (2016).

91. Zhang, X. C. Change of Glutamate Metabolism in the Brain of CUMS Rats: A Comparison of Electro-Acupuncture and Manul Acupuncture on Siguan acupoints(PhD. Thesis, in Chinese). Available at: http://www.cnki.net/KCMS/detail/detail. aspx?FileName $=1016208712 . n h \& D b N a m e=C D F D 2016$ (Accessed 13th Jan 2017) (2016).

92. Zhang, X. H. Effects of Acupuncture in Regulating the Expression of Brain-Derived Neurophic through Epigenetics (DNA Histone Acetylation) and PKA Signaling Pathway (PhD. Thesis, in Chinese). Available at: http://www.cnki.net/KCMS/detail/detail. aspx?FileName $=1016069877 . n h \& d b n a m e=C D F D L A S T 2016$ (Accessed 13th Jan 2017) (2016).

93. Zhao, S. L. Clinical and Experimental Study on Electro-Acupuncture in the Treatment of Depression(PhD. Thesis, in Chinese). Available at: http://www.cnki.net/KCMS/detail/detail.aspx?FileName=2005095936.nh\&DbName=CDFD2005 (Accessed 5th Aug 2015) (2005).

94. Zheng, Z. Y., Xu, B. J., Zhou, L. Y. \& Wu, M. J. Influences of Scalp Acupuncture On CRF Expression of Hypothalamus in Depression Model Rats. Journal of Clinical Acupuncture and Moxibustion. 29, 61-63 (2013).

95. Zhou, S. H. Effects of Acupuncture On Behavior Changes of Depressed Model Rats. Lishizhen Medicine and Materia Medica. Research. 19, 116-118 (2008).

96. Zhu, X. C. et al. Effect of Electroacupuncture Stimulation of "Baihui"(GV20),Etc.On Changes of Behavior and Plasma Ghrelin Content in Depression Rats. Acupuncture. Research. 40, 283-289 (2015).

97. Zhuang, L. Y., Xu, S. F. \& Tang, C. Z. Effects of Different Intervention Methods on Behavior Changes in Depression Rats. Acupuncture. Research. 32, 93-97 (2007).

98. Xu, J. et al. NMR-based metabolomics Reveals Alterations of Electro-acupuncture Stimulations on Chronic Atrophic Gastritis Rats. Sci Rep. 7, 45580 (2017).

99. Willner, P. Validity, Reliability and Utility of the Chronic Mild Stress Model of Depression: A 10-year Review and Evaluation. Psychopharmacology. 134, 319-329 (1997).

100. De Vry, J. \& Schreiber, R. The Chronic Mild Stress Depression Model: Future Developments from A Drug Discoveryperspective. Psychopharmacology. 134, 349-350 (1997).

101. Smith, C. A., Hay, P. P. \& Macpherson, H. Acupuncture for Depression. The Cochrane database of systematic reviews. D4046 (2010).

102. He, W., Zhao, X., Li, Y., Xi, Q. \& Guo, Y. Adverse Events Following Acupuncture: A Systematic Review of the Chinese Literature for the Years 1956-2010. Journal of alternative and complementary medicine (New York, N.Y.). 18, 892-901 (2012).

103. Tsukayama, H., Yamashita, H., Kimura, T. \& Otsuki, K. Factors that influence the applicability of sham needle in acupuncture trials: two randomized, single-blind, crossover trials with acupuncture-experienced subjects. Clinical Journal of Pain. 22, 346-349 (2006).

104. Kilkenny, C., Browne, W. J., Cuthill, I. C., Emerson, M. \& Altman, D. G. Improving Bioscience Research Reporting: The ARRIVE Guidelines for Reporting Animal Research. Journal of pharmacology \& pharmacotherapeutics. 1, 94-99 (2010).

105. Macleod, M. R., O'Collins, T., Howells, D. W. \& Donnan, G. A. Pooling of Animal Experimental Data Reveals Influence of Study Design and Publication Bias. Stroke; a journal of cerebral circulation. 35, 1203-1208 (2004).

\section{Acknowledgements}

We would like to express our gratitude to Xin Cao (Department of Pharmacology, Faculty of Medicine, Toho University) and Ye Hong (Southwestern University of Finance and Economics) for English edit. This work was supported by grants from the National Natural Science Foundation of China (No.81202743, 81403479, 81303019, 81574062), Natural Science Foundation of JiangSu Province (No. BK20151569) and Qing Lan Project of JiangSu Province (2016). The funders had no role in study design, data collection and analysis, decision to publish, or preparation of the manuscript. 


\section{Author Contributions}

All the authors have contributed to this article. Study concept and design: R.Z.K., H.C. and S.F.L. Acquisition of data: R.Z.K., H.C. Analysis and interpretation of data: M.L.Y and S.F.L. Production chart: R.Z.K., H.C., T.C.X. and S.P.F. Wrote the paper: R.Z.K. and H.C. Revised the manuscript: SFL. All the authors have reviewed the manuscript.

\section{Additional Information}

Supplementary information accompanies this paper at doi:10.1038/s41598-017-09712-1

Competing Interests: The authors declare that they have no competing interests.

Publisher's note: Springer Nature remains neutral with regard to jurisdictional claims in published maps and institutional affiliations.

(c) (i) Open Access This article is licensed under a Creative Commons Attribution 4.0 International License, which permits use, sharing, adaptation, distribution and reproduction in any medium or format, as long as you give appropriate credit to the original author(s) and the source, provide a link to the Creative Commons license, and indicate if changes were made. The images or other third party material in this article are included in the article's Creative Commons license, unless indicated otherwise in a credit line to the material. If material is not included in the article's Creative Commons license and your intended use is not permitted by statutory regulation or exceeds the permitted use, you will need to obtain permission directly from the copyright holder. To view a copy of this license, visit http://creativecommons.org/licenses/by/4.0/.

(c) The Author(s) 2017 